\title{
Magnetically stabilized nematic order: Three-dimensional bipartite optical lattices
}

\author{
F. Zhou, ${ }^{1}$ M. Snoek, ${ }^{2}$ J. Wiemer, ${ }^{2}$ and I. Affleck ${ }^{1}$ \\ ${ }^{1}$ Department of Physics and Astronomy, University of British Columbia, 6224 Agriculture Road, Vancouver, \\ British Columbia, Canada V6T $1 Z 1$ \\ ${ }^{2}$ Institute for theoretical physics, Utrecht University, Leuvenlaan 4, 3584 CE Utrecht, The Netherlands
}

(Received 2 August 2004; published 24 November 2004)

\begin{abstract}
We study magnetically stabilized nematic order for spin-one bosons in optical lattices. We show that the Zeeman field-driven quantum phase transitions between non-nematic Mott states and quantum spin nematic Mott states in the weak hopping limit are in the universality class of the ferromagnetic $X X Z(S=1 / 2)$ spin model. We further discuss these transitions as condensation of interacting magnons. The development of $\mathrm{O}(2)$ nematic order when external fields are applied corresponds to condensation of magnons, which breaks a U(1) symmetry. Microscopically, this results from a coherent superposition of two non-nematic states at each individual site. Nematic order and spin-wave excitations around critical points are studied and critical behaviors are obtained in a dilute gas approximation. We also find that spin-singlet states are unstable with respect to quadratic Zeeman effects and Ising nematic order appears in the presence of any finite quadratic Zeeman coupling. All discussions are carried out for states in three-dimensional bipartite lattices.
\end{abstract}

DOI: 10.1103/PhysRevB.70.184434

PACS number(s): 03.75.Mn, 05.30.Jp, 75.10.Jm

\section{INTRODUCTION}

When the dimensionless exchange coupling strength is strong enough, Mott states of spinful particles are known to develop certain spin order. In the opposite limit, quantum fluctuations usually restore the broken symmetry resulting in spin-singlet states. This widely accepted belief, however, does not exclude, and furthermore implies certain "hidden" fluctuating order in the symmetry restored states. The preexisting order appears dynamically at certain time and length scales and is in general very relevant to the low-energy physics. Particularly if an external magnetic field is applied, nontrivial spin (magnetic) order might be induced because of the coupling between magnetic excitations and external fields. In other words, an external field can stabilize spin order in a parameter regime where order is absent in zero field.

The possible field-induced ordering usually results from condensation of magnetic excitations or magnons. The fieldinduced quantum phase transitions between states of different magnetic correlations and possible magnetically stabilized order close to critical points can be investigated by examining magnon excitations in one of the phases involved in the phase transitions.

A well-known example is the $S=1$ antiferromagnetic spin chain. ${ }^{1-7}$ The ground state is the spin singlet AKLT state and all spin excitations are fully gapped by the Haldane gap. An applied Zeeman field along the positive $z$ direction, although it has zero coupling with the singlet ground state, couples to spin excitations, and lowers the energy of excitations in the $S=1, S_{z}=1$ branch because of the Zeeman coupling. At critical fields, the zero momentum excitation becomes degenerate with the spin-singlet ground state signifying a quantum phase transition.

The presence and nature of induced canted Néel order in this case therefore depend crucially on interactions between magnetic excitations in the AKLT phase. One can easily visualize that the transition is of first order if the interactions of condensed magnons are attractive or absent. Naturally, the magnetization per lattice site in this case jumps by a finite value at the critical point as a result of condensation; furthermore the canted Néel order would not appear in this case. The phase transition would be simply between a fully polarized state and a spin-singlet state.

However, if interactions between magnons are repulsive, condensation takes place continuously because of finite chemical potentials for repulsively interacting magnons. Thus the magnetization per site, which is proportional to the density of magnons varies continuously across the critical point and the transition is of second order. In this case, the resultant state has canted Néel order. For the $S=1$ antiferromagnetic spin chain, numerical results show that the magnetization indeed varies continuously and imply that magnons have repulsive interactions. ${ }^{7}$ In fact, at higher magnetic fields, external fields do induce canted Néel order in $S=1$ spin chains (only quasi-long-range order prevails in chains). Condensation of magnons has also been recently studied in three-dimensional frustrated magnets (see, for instance, Refs. 8 and 9).

Therefore, to investigate magnetically stabilized order, it is important to understand interactions between magnons or magnetic excitations. Generally, microscopic calculations of magnon interactions are not only very difficult but also practically impossible for low-spin systems because of the uncontrollable approximations involved. However, in the case we are going to examine we do evaluate the interactions microscopically in various situations; therefore we believe the results about magnetically stabilized quantum spin nematic order and quantum phase transitions are precise in this sense. We also want to emphasize that magnon condensation which interests us in this article occurs in both high dimensional and one-dimensional nonfrustrated optical lattices. The one-dimensional limit will be treated in a separated paper.

The purpose of this article is to understand the magnetically stabilized nematic order of spin-one bosons in optical lattices. As emphasized above, our starting point will be a 
series of Mott states with no nematic order, some of which also have zero coupling with external (linear) Zeeman fields. Our main subject is to investigate the development of nematic order or spontaneous symmetry breaking in the $x y$ plane when external magnetic fields are applied along the $z$ direction. In particular, we will focus on the nematic order close to critical points where our results are actually exact. Furthermore, the approach we employ here is believed to yield exact phase boundaries between nematic states and nonnematic Mott states, a rather remarkable conclusion thanks to the powerful mapping developed below.

Spin-correlated Mott states for spin-one bosons have recently attracted considerable interest. ${ }^{10-14}$ Theoretical works indicate that spin correlations in Mott states depend on three dimensionless parameters. ${ }^{11,12}$ The first one is the dimensionless exchange coupling $\eta\left(=J_{\mathrm{ex}} / E_{s}\right)$ which is defined as the ratio between the exchange interaction $J_{\text {ex }}$ and the bare spin gap for an individual site $E_{s}$. The second parameter is the parity $P$ (even or odd) of the number of particles per site in Mott states. The last parameter $D$ is the dimensionality of optical lattices (we assume all lattices are bipartite).

For $D=2,3$, it was argued that Mott states for all odd $P$ are nematically ordered. In fact irrespective of the exact number of particles per site in this case, the effective Hamiltonian in the small hopping limit $(\eta \ll 1)$ is equivalent to the bilinear-biquadratic model for $S=1$ spin chains. ${ }^{14}$ For even $P$ on the other hand, nematic and spin singlet Mott states are present for large $\eta$ and small $\eta$ limits, respectively. The onedimensional physics $(D=1)$ is dominated by quantum fluctuations. Both dimerized valence bond crystals and nondegenerate spin-singlet states have been found. As a result of symmetry restoring, the low-energy dynamics in Mott states is mapped into the even- and odd-class quantum dimer models. ${ }^{11}$ Furthermore, atoms have a tendency to be fractionalized into solitonic elementary excitations in this limit. Superfluid phases have distinct topological properties and remain to be fully understood.

Responses of correlated states of spin-one bosons to external fields are fascinating. For nematic condensates, the responses are continuous. The linear coupling between condensates and weak external fields pins the easy axis in the $x y$ plane perpendicular to external fields, and $\mathrm{O}(3)$ nematic condensates become $\mathrm{O}(2)$ ones, or canted nematic states; quadratic coupling, however, pins the nematic easy axis along the direction of coupling. ${ }^{15}$ For spin singlet condensates, the magnetization jumps discontinuously as a result of a series of level crossings between states $\left|S, S_{z}=S\right\rangle$ and $\left|S+2, S_{z}=S+2\right\rangle$ (Ref. 16) (also see general discussions about condensates in Refs 17 and 18). Responses of Mott states to external Zeeman fields and various transitions have recently been studied in a mean-field approach. Mott states can either respond to external fields continuously similar to nematic condensates or develop interesting magnetization plateaus similar to charge quantization in a Mott state. ${ }^{19}$

In this article, we study magnetically stabilized nematic order in optical lattices. Particularly we demonstrate the development of nematic order as repulsively interacting magnons in non-nematic Mott states condense. We investigate the induced nematic order associated with the spontaneous breaking of $\mathrm{O}(2)$ nematic symmetry, the magnetization and the spin-wave velocity.
In Sec. II, we review the properties of spin-singlet Mott states and introduce a projected nematic order parameter for discussions on spin partially polarized states. For a given lattice site with two particles, we show that nematic order can be established if a spin-singlet state is in a superposition with a higher spin state $\left|S=2, S_{z}=2\right\rangle$. The relative phase between these two states in the superposition determines the easy axis of the nematic order parameter, or the orientation of spin-nematic states. In Sec. III, we study the general characterization of nematic order in spin-polarized Mott states; we propose a projected nematic order parameter which projects away trivial contributions from spin polarization.

In Sec. IV, we truncate the Hilbert space close to critical magnetic fields and show that the resultant Hamiltonian is an $X X Z(S=1 / 2)$ pseudospin model in an effective field along the $z$ direction. We carry out microscopic calculations of all parameters in the effective ferromagnetic $X X Z$ model. These calculations are done for two particles per site, four particles per site, and in the large $N$ (even) limit. The quantum rotor model studied in previous works is employed to facilitate calculations.

In Sec. V, close to critical lines and a tricritical point we further study the properties of various phases of the $X X Z$ model in both the semiclassical approximation and dilute gas approximation based on the Holstein-Primakov boson representation. We analyze the interactions between HolsteinPrimakov bosons or magnons. We obtain the exact phase boundaries for the ferromagnetic $X X Z$ model by investigating the instability lines of magnon excitations. We also discuss the relation between condensation of magnons close to critical lines, the variation of magnetization across critical points, and the appearance of ferromagnetic order.

In Sec. VI, we investigate, in details, the development of magnetically stabilized nematic order by examining results following the mapping to the $X X Z$ model and to the Holstein-Primakov condensation problem. We notice that results about phase boundaries, nematic order, and spin-wave velocities in the critical regime can be obtained in a dilute gas approximation and therefore, remarkably, are exact. In Sec. VII, we further study the effect of quadratic Zeeman coupling. Finally, in Sec. VIII, we conclude our investigation on this subject.

\section{SPIN SINGLET MOTT STATES, FLUCTUATING NEMATIC ORDER, AND PROJECTED NEMATIC ORDER}

The Hamiltonian for spin-one bosons with antiferromagnetic interactions in optical lattices in an external field can be conveniently expressed as ${ }^{10-14,19}$

$$
\begin{aligned}
H= & E_{s} \sum_{k} \hat{\mathbf{S}}_{k}^{2}+E_{c} \sum_{k} \hat{\rho}_{k}^{2}-\mu_{0} \sum_{k} \hat{\rho}_{k}-\sum_{k} \hat{\mathbf{S}}_{k z} H_{z} \\
& -t \sum_{\langle k l\rangle}\left(\psi_{k \alpha}^{\dagger} \psi_{l \alpha}+\text { H.c. }\right) .
\end{aligned}
$$

Here $\psi_{k \alpha}^{\dagger}\left(\psi_{k \alpha}\right), \alpha=x, y, z$ are creation (annihilation) operators for spin-one particles in three different states at site $k . \hat{\mathbf{S}}_{k \alpha}$ $=-i \epsilon_{\alpha \beta \gamma} \psi_{k \alpha}^{\dagger} \psi_{k \beta}$ and $\hat{\rho}_{k}=\psi_{k \alpha}^{\dagger} \psi_{k \alpha}$ are the spin and number operators defined for each lattice site. It is easy to verify that 
$\left[\hat{S}_{k \alpha}, \hat{S}_{k^{\prime} \beta}\right]=i \delta_{k k^{\prime}} \epsilon_{\alpha \beta} \hat{S}_{k \gamma} E_{c, s}$ are "bare" charge and spin gaps studied in Ref. 11 and $\mu_{0}$ is the chemical potential. Finally, the sum over $\langle k l\rangle$ represents the sum over all neigboring sites.

In Mott states, the Hamiltonian can be reduced to the following effective one:

$$
\begin{gathered}
H=E_{s} \sum_{k} \hat{\mathbf{S}}_{k}^{2}-\sum_{k} \hat{\mathbf{S}}_{k z} H_{z}-J_{\mathrm{ex}} \sum_{\langle k l\rangle}\left[\hat{Q}_{\alpha \beta}(k) \hat{Q}_{\beta \alpha}(l)+\text { H.c. }\right], \\
\hat{Q}_{\alpha \beta}(k)=\psi_{k \alpha}^{\dagger} \psi_{k \beta}-\frac{1}{3} \delta_{\alpha \beta} \psi_{k \gamma}^{\dagger} \psi_{k \gamma} .
\end{gathered}
$$

Equation (2) is valid when $t \ll E_{c}$ and $E_{s} \ll E_{c} . J_{\mathrm{ex}}=t^{2} / 2 E_{c}$ is the exchange interaction.

The effective Hamiltonian with zero Zeeman coupling was obtained in a few previous works; solutions to this Hamiltonian have been studied in various limits. When the external field is absent and $\eta=J_{\mathrm{ex}} / E_{s}$ is much less than unity, the ground state is a spin singlet for an even number of particles per site $(N)$. The spin-singlet Mott (SSM) ground state in this limit is the product of spin singlets at each individual site (up to a normalization factor) ${ }^{11,13}$

$$
\Psi_{\mathrm{SSM}}=\prod_{k} \frac{\left(\psi_{k \alpha}^{\dagger} \psi_{k \alpha}^{\dagger}\right)^{N / 2}}{\sqrt{(N+1) !}}|\mathrm{vac}\rangle
$$

Meanwhile, the spin fully polarized (SFP) ground state is the product of on-site maximally polarized states

$$
\Psi_{\mathrm{SFP}}=\prod_{k} \frac{\left(\psi_{k x}^{\dagger}+i \psi_{k y}^{\dagger}\right)^{N}}{\sqrt{2^{N} N !}}|\mathrm{vac}\rangle .
$$

In SSM states, $\left\langle\hat{Q}_{\alpha \beta}(k)\right\rangle=0$ as a result of the rotational invariance of the wave function and thus there is no nematic order. The hidden fluctuating nematic order can be studied by examining higher moments. For $N=2$, one can easily obtain the following results:

$$
\begin{aligned}
& \left\langle\hat{Q}_{\alpha^{\prime} \beta^{\prime}}(k) \hat{Q}_{\alpha \beta}\left(k^{\prime}\right)\right\rangle \\
& \quad=\frac{2}{3} \delta_{k k^{\prime}}\left(\delta_{\alpha^{\prime} \beta} \delta_{\beta^{\prime} \alpha}+\delta_{\alpha^{\prime} \alpha} \delta_{\beta \beta^{\prime}}-\frac{2}{3} \delta_{\alpha^{\prime} \beta^{\prime}} \delta_{\alpha \beta}\right),
\end{aligned}
$$

which indicate on-site fluctuating nematic order. More explicitly, one finds the amplitude of fluctuations of nematic tensor matrix elements

$$
\left\langle\left[\hat{Q}_{\alpha \beta}(k)\right]^{2}\right\rangle=\frac{2}{3}\left(1+\frac{1}{3} \delta_{\alpha \beta}\right) .
$$

To investigate the responses of spin singlet Mott states or other non-nematic states, which exhibit certain fluctuating nematic order, to external fields, it is important to understand how nematic order can be induced by external Zeeman fields. For this purpose, we focus on the simplest situation where $J_{\mathrm{ex}}$ is zero and treat each site independently. We would like to demonstrate the following important statement: nematic order appears whenever a spin-singlet state and a polarized state are in a linear superposition.

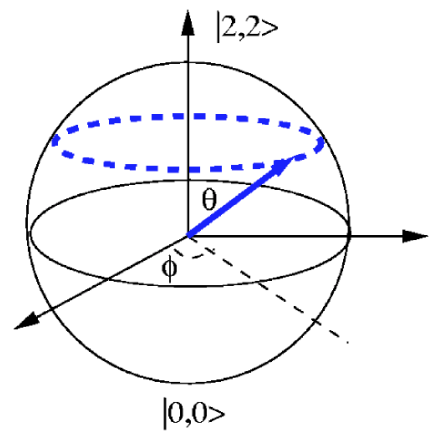

FIG. 1. (Color online) Coherent states $|\boldsymbol{\Omega}\rangle$ at the Bloch sphere of pseudospins. All states except the north pole $(\theta=0)$ and south pole $(\theta=\pi)$ ones have nonvanishing expectation value of nematic tensor operator $\hat{Q}_{\alpha \beta}$.

We first consider two particles at one lattice site. The Hilbert space is spanned by fivefold degenerate $S=2$ states and a spin-singlet state. When an external field is applied along the $z$ direction, the fivefold degeneracy is completely lifted while the maximally polarized state $\left|S=2, S_{z}=2\right\rangle$ approaches the spin-singlet ground state. When the level crossing takes place, the spin projection along the $z$ direction jumps by $2 \hbar$. It is obvious that no nematic order is induced in this simple limit and there are no transitions between nematic states and spin-singlet states.

However, at the level crossing points, one can further study the properties of coherent superposition of $|0,0\rangle$ and $|2,2\rangle$ states while the rest of states are highly excited ones at these crossings. Let us introduce a coherent state defined in the two-state subspace as

$$
\begin{gathered}
|\boldsymbol{\Omega}\rangle=\cos \frac{\theta}{2} \exp \left(-i \frac{\phi}{2}\right)|\uparrow\rangle+\sin \frac{\theta}{2} \exp \left(i \frac{\phi}{2}\right)|\downarrow\rangle ; \\
|\uparrow\rangle=\left|S=2, S_{z}=2\right\rangle=\frac{1}{2 \sqrt{2}}\left(\psi_{x}^{\dagger}+i \psi_{y}^{\dagger}\right)^{2}|\mathrm{vac}\rangle, \\
|\downarrow\rangle=\left|S=0, S_{z}=0\right\rangle=\frac{1}{\sqrt{6}} \psi_{\alpha}^{\dagger} \psi_{\alpha}^{\dagger}|\mathrm{vac}\rangle .
\end{gathered}
$$

Here the unit vector is defined as $\boldsymbol{\Omega}$ $=(\sin \theta \cos \phi, \sin \theta \sin \phi, \cos \theta)$. One can easily verify that

$$
\left\langle\hat{S}_{z}\right\rangle=2 \hbar \cos ^{2} \frac{\theta}{2} .
$$

$S_{z}$ reaches the maximum when $\theta=0$ and the minimum when $\theta=\pi / 2$.

Direct calculations of the usual nematic order parameter $Q_{\alpha \beta}$ defined as the expectation value of the tensor operator $\hat{Q}_{\alpha \beta}$ in Eq. (2) suggest that it have a nontrivial structure in the maximally polarized state (see Fig. 1). Namely, it contains (a) an antisymmetric tensor as a result of spin polarization and (b) a traceless symmetric part which reflects the explicit rotational symmetry breaking by the magnetic field but is not associated with the spontaneous nematic symmetry breaking in a plane perpendicular to the polarization. 
To discuss the nematic order in fully or partially polarized states, it is therefore essential to introduce a projected nematic order parameter $Q_{\alpha \beta}^{P}(\alpha, \beta=x, y, z)$

$$
Q_{\alpha \beta}^{P}=Q_{\alpha \beta}-\left(Q_{\alpha^{\prime} \beta^{\prime}} \Pi_{\beta^{\prime} \alpha^{\prime}}^{1}\right) \Pi_{\alpha \beta}^{1}-\left(Q_{\alpha^{\prime} \beta^{\prime}} \Pi_{\beta^{\prime} \alpha^{\prime}}^{2}\right) \Pi_{\alpha \beta}^{2} .
$$

Note that in the projected order parameter, the component associated with the nematic symmetry breaking remains while the components associated with polarization have been projected away. Two tensors we would like to project away are defined as

$$
\begin{gathered}
\Pi_{\alpha \beta}^{1}=\frac{1}{\sqrt{2}}\left(\begin{array}{ccc}
0 & -i & 0 \\
i & 0 & 0 \\
0 & 0 & 0
\end{array}\right) . \\
\Pi_{\alpha \beta}^{2}=\frac{1}{\sqrt{6}}\left(\begin{array}{ccc}
-1 & 0 & 0 \\
0 & -1 & 0 \\
0 & 0 & 2
\end{array}\right) .
\end{gathered}
$$

One finds that the nematic order only appears in the $x y$ plane perpendicular to the external fields. It is indeed straightforward to show that the truncation of $Q_{\alpha \beta}^{P}$ in the $x y$ plane (i.e., elements with $\alpha=x, y$ only) for a coherent state defined above is

$$
Q_{\alpha \beta}^{P x y}=\frac{1}{\sqrt{3}} \sin \theta\left(\begin{array}{cc}
\cos \phi & \sin \phi \\
\sin \phi & -\cos \phi
\end{array}\right) .
$$

Two important features in Eq. (11) are worth emphasizing. First, the two eigenvalues correspond to $\pm \sin \theta / \sqrt{3}$ and are proportional to the coherence factor in the linear superposition of coherent states. They are nonvanishing only if $\theta$ is not zero or $\pi$. Therefore nontrivial nematic order always appears when $|\uparrow\rangle$ and $|\downarrow\rangle$ two states are in a linear superposition.

Secondly, the eigenvector with the maximal eigenvalue represents the easy axis of nematic order. And the easy axis is fully characterized by the azimuthal angle of $\boldsymbol{\Omega}$. Indeed, one finds that the easy axis in the $x y$ plane is defined as a 2D unit vector in the $x y$ plane:

$$
\omega=\left(\cos \frac{\phi}{2}, \sin \frac{\phi}{2}\right) .
$$

When $\phi$ varies from 0 to $2 \pi$, the easy axis $\omega$ rotates by $\pi$ angle in the $x y$ plane. And the nematic order parameter is indeed a tensor constructed out of the 2D unit vector $\omega$

$$
Q_{\alpha \beta}^{P x y}=\frac{2}{\sqrt{3}} \sin \theta\left(\omega_{\alpha} \omega_{\beta}-\frac{1}{2} \delta_{\alpha \beta}\right) .
$$

\section{NEMATIC ORDER PARAMETER FOR SPIN PARTIALLY POLARIZED STATES: GENERAL CHARACTERIZATION}

As we have already seen in the previous section, the complication of characterizing nematic order when spins are partially polarized comes from the explicit symmetry breaking induced by external fields. So in this case one has to deal with the tensor $\hat{Q}_{\alpha \beta}$ which has nontrivial elements even without nematic order. To distinguish the spontaneous symmetry breaking due to the formation of nematic order from explicit symmetry breaking in the presence of polarization, special care needs to be taken of the elements which are induced by spin polarization.

A general scheme to project out the nematic order parameter tensor appears to be possible in a way, similar to what was carried out in the previous section. Assume spins are polarized along direction $\mathbf{s}$ (unit vector). Introducing two projection tensors

$$
\Pi_{\alpha \beta}^{1}=\frac{1}{\sqrt{2}} i \epsilon_{\alpha \beta \gamma} \mathbf{s}_{\gamma}, \Pi_{\alpha \beta}^{2}=\frac{3}{\sqrt{6}}\left(\mathbf{s}_{\alpha} \cdot \mathbf{s}_{\beta}-\frac{1}{3} \delta_{\alpha \beta}\right),
$$

we again are able to define a projected nematic order parameter as in Eq. (9).

When the nematic symmetry is broken along the direction $\omega$ (unit vector), in the large $N$ limit one can easily demonstrate that $\mathbf{s} \cdot \omega=0$ following the algebras in Refs. 15 and 11; and $\omega$ and $\mathbf{s}$ always appear to be orthogonal. One can further define

$$
\mathbf{m}=\mathbf{s} \times \omega .
$$

Then $\omega, \mathbf{m}$, and $\mathbf{s}$ form an orthogonal triad.

It is possible to verify the validity of the definition for nematic order parameters given Eqs. (9) and (14). For instance, one can consider the following spin partially polarized nematic states:

$$
|\Psi\rangle=\frac{\left[\left(\cos \frac{\xi}{2} \mathbf{n}+i \sin \frac{\xi}{2} \mathbf{m}\right) \psi_{\alpha}^{\dagger}\right]^{N}}{\sqrt{N !}}|\mathrm{vac}\rangle .
$$

We have assumed that $\mathbf{n}$ and $\mathbf{m}$ are orthogonal, i.e., $\mathbf{n} \cdot \mathbf{m}$ $=0$; $\xi$ varies from 0 to $\pi$. Following the discussions in Refs. 15,11 , and 13, states specified in Eq. (16) with $\xi=0$ form a complete set of $N$-particle-condensate wave functions. And a condensate with total spin $S, S_{z}(S \leqslant N)$ can be expressed in terms of spherical harmonics $Y_{S, S_{z}}(\mathbf{n})$ in this representation.

When $\xi \neq 0$, a state given above is polarized along a direction perpendicular to $\mathbf{n}$ and $\mathbf{m}$. Indeed,

$$
\langle\hat{\mathbf{S}}\rangle=N \sin \xi \mathbf{n} \times \mathbf{m}, \quad \mathbf{s}=\mathbf{n} \times \mathbf{m} .
$$

A direct calculation shows that the projected nematic order parameter can be expressed in terms of three orthogonal unit vectors $(\mathbf{n}, \mathbf{m}, \mathbf{s})$,

$$
\frac{Q_{\alpha \beta}^{P}}{N}=\cos ^{2} \frac{\xi}{2} n_{\alpha} n_{\beta}+\sin ^{2} \frac{\xi}{2} m_{\alpha} m_{\beta}+\frac{1}{2} s_{\alpha} s_{\beta}-\frac{1}{2} \delta_{\alpha \beta} .
$$

Note that the projected nematic order parameter is traceless and fully symmetric. ${ }^{20}$ One can further truncate the projected matrix in the $(\mathbf{n}, \mathbf{m})$ plane perpendicular to $\mathbf{s}$ and indeed find that 


$$
Q_{\alpha \beta}^{P n m}=\frac{N}{2}\left(\begin{array}{cc}
\cos \xi & 0 \\
0 & -\cos \xi
\end{array}\right)
$$

which is diagonal when $\alpha, \beta$ are chosen to be along the axis n or $\mathbf{m}$.

The projected nematic order parameters $Q_{\alpha \beta}^{P}, Q_{\alpha \beta}^{P n m}$ vanish when spins are fully polarized or $\xi=\pi / 2$ and the nematic matrix has zero eigenvalues. When $\xi \neq \pi / 2$, the matrix has nontrivial eigenvalues $\pm N / 2 \cos \xi$. The nematic axis $\omega$ therefore lies along the direction of $\mathbf{n}$ when $\xi$ varies from 0 to $\pi / 2$ and along the direction of $\mathbf{m}$ when from $\pi / 2$ to $\pi$. The nematic matrix eigenvalues reach maxima when $\xi=0$ or $\pi$, representing spin unpolarized nematic states.

Obviously, nematic symmetry order can develop along an arbitrary direction in a plane perpendicular to $\mathbf{s}$. In fact, an $\mathrm{O}(2)$ rotation of the orthogonal basis $(\mathbf{n}, \mathbf{m})$ along $\mathbf{s}$ by a $\phi$ angle, while leaving $\langle\hat{\mathbf{S}}\rangle$ invariant, results in a new nematic state with easy axis $\omega$ :

$$
\omega=\cos \phi \mathbf{n}+\sin \phi \mathbf{m}
$$

if $\xi \in[0, \pi / 2]$, and

$$
\omega=-\sin \phi \mathbf{n}+\cos \phi \mathbf{m}
$$

if $\xi \in[\pi / 2, \pi]$.

In terms of the easy axis $\omega$, the projected order parameter can be conveniently expressed as

$$
Q_{\alpha \beta}^{P n m}=N|\cos \xi|\left(\omega_{\alpha} \omega_{\beta}-\frac{1}{2} \delta_{\alpha \beta}\right) .
$$

To summarize, we have shown that a projected traceless nematic tensor order parameter should be introduced to study nematic ordering in the presence of external fields.

\section{FERROMAGNETIC $X X Z$ MODEL AS THE EFFECTIVE HAMILTONIAN CLOSE TO CRITICAL POINTS}

\section{A. Phenomenology}

To study the magnetically stabilized nematic order, we consider a limit when the exchange interaction $J_{\text {ex }}$ is much less than $E_{s}$. For an even number of particles per site and in the absence of external fields, the ground state is a spinsinglet Mott state and nematic order is absent. The development of nematic order first occurs when

$$
2 H_{z} \approx 6 E_{s} \gg J_{\text {ex }} .
$$

The Hilbert space for the whole lattice is a direct product of spin towers $\mathcal{H}_{k}$ at each site:

$$
\mathcal{H}_{T 0}=\mathcal{H}_{1} \otimes \mathcal{H}_{2} \otimes \mathcal{H}_{3} \otimes \cdots \otimes \mathcal{H}_{k} \otimes \cdots .
$$

The on-site Hilbert space $\mathcal{H}_{k}$ is spanned by $(N+1)(N+2) / 2$ states, with spins equal to $0,2,4, \ldots, N$; the dimension of the Hilbert space for the whole lattice is

$$
\mathcal{D}_{T 0}=\left(\frac{(N+1)(N+2)}{2}\right)^{V_{T}},
$$

where $V_{T}$ is the number of lattice sites.
When the external fields satisfying the condition in Eq. (23) are applied and when $J_{\mathrm{ex}}=0$, at each individual site the first excited state $\left|S=2, S_{z}=2\right\rangle$ and ground state $\left|S=0, S_{z}=0\right\rangle$ are nearly degenerate and are far away from other excited states. At the point when the field reaches a value so that

$$
H_{z}=3 E_{s},
$$

level crossing between the the ground state and first excited state occurs as mentioned briefly in the previous section. Following the discussions in Sec. II, if the hopping or the exchange energy is set to be precisely zero, then magnetization jumps and $Q_{\alpha \beta}^{P}=0$. In this case, nematic order is not induced by external fields.

As shown in the previous section, for nematic order to be present, two nearly degenerate states have to be in a linear superposition. In this sense, it is the exchange process in the vicinity of level crossing points which eventually naturally leads to nematic order which does not exist in zero fields. This observation leads us to truncate the on-site Hilbert space into a two-dimensional one for a pseudospin. The truncated Hilbert space for the whole lattice is then a product of pseudospin Hilbert space $\mathcal{S}_{k}$ at each site $k$,

$$
\mathcal{H}_{T t}=\mathcal{S}_{1} \otimes \mathcal{S}_{2} \otimes \mathcal{S}_{3} \cdots \otimes \mathcal{S}_{k} \otimes \cdots
$$

and the on-site pseudospin Hilbert space $\mathbf{S}_{k}$ consists of two states

$$
|\uparrow\rangle=\left|S=2, S_{z}=2\right\rangle,|\downarrow\rangle=\left|S=0, S_{z}=0\right\rangle .
$$

For two particles per site, the microscopic wave function of these two states is given in Eq. (7).

The dimension of the truncated space $\mathcal{D}_{T t}$ is exponentially small compared with the original one $\mathcal{D}_{T 0}$, i.e.,

$$
\mathcal{D}_{T t}=2^{V_{T}} \ll \mathcal{D}_{T 0} .
$$

It is also independent of the number of particles per site. The phenomenology for different even numbers of particles per site is therefore identical.

The hopping between two nearest neighbors in lattices introduces exchange interactions between pseudospins. We will present results of microscopic calculations in the following subsection. Here we provide a phenomonology of this model. To facilitate discussions, we define $|\uparrow\rangle$ and $|\downarrow\rangle$ to be two eigenstates of Pauli matrix $\sigma_{z}$,

$$
\sigma_{z}|\uparrow\rangle=|\uparrow\rangle, \sigma_{z}|\downarrow\rangle=-|\downarrow\rangle .
$$

Note that these two pseudospins are also eigenstates of the spin operator $\hat{\mathcal{S}}_{z}$. Therefore the pseudospin algebra corresponds to the projection of the usual SU(2) spin algebra in the truncated pseudospin space. For instance, one can verify the following mapping:

$$
\hbar\left(\sigma_{z}+1\right) \rightarrow \hat{\mathbf{S}}_{z}, \hbar \sigma^{+} \rightarrow \hat{\mathbf{S}}^{+}, \hbar \sigma^{-} \rightarrow \hat{\mathbf{S}}^{-}
$$

An important and obvious fact is that single-particle hopping conserves the total spin of two sites and its projection along all directions including the $z$ direction. Following Eq. (31), this conservation of spins implies that any induced exchange coupling have to as well conserve the pseudospin defined along $\sigma_{z}$ axis in the presence of external Zeeman 
fields. Furthermore, the superexchange due to virtual hopping between two bosonic $S=1 / 2$ pseudospins results in a ferromagnetic coupling which is to be further verified by microscopic calculations.

Based on the above considerations, one concludes that the effective Hamiltonian in the truncated space should be

$$
\begin{aligned}
\frac{H_{\mathrm{eff}}}{J_{\mathrm{ex}}}= & -2 \epsilon_{0} \sum_{\langle k l\rangle}\left(\sigma_{k}^{+} \sigma_{l}^{-}+\sigma_{k}^{-} \sigma_{l}^{+}\right) \\
& -(\beta+1) \epsilon_{0} \sum_{\langle k l\rangle} \sigma_{k z} \sigma_{l z}-\epsilon_{0} h_{z} \sum_{k} \sigma_{k z} .
\end{aligned}
$$

Here $\epsilon_{0}, \beta$ depends on microscopic details of states and should be a function of the number of particles per site and $\eta_{1}$, the ratio between $E_{s}$ and $E_{c} . h_{z}$ further depends on $\eta$ (the ratio between $J_{\text {ex }}$ and $E_{s}$ ) and the ratio between external fields $H_{z}$ and $J_{\mathrm{ex}}$.

One can easily recast the Hamiltonian into the following ferromagnetic $X X Z$ model in an effective external field along the $z$ direction,

$$
\frac{H_{X X Z}}{\epsilon_{0} J_{\mathrm{ex}}}=-\sum_{\langle k l\rangle} \sigma_{k \alpha} \sigma_{l \alpha}-\beta \sum_{\langle k l\rangle} \sigma_{k z} \sigma_{l z}-h_{z} \sum_{k} \sigma_{k z} .
$$

Because external magnetic fields are applied along the $z$ direction, with the particular choices of eigenstates for the pseudospin Pauli matrix $\sigma_{z}$ in Eq. (30), the Hamiltonian in Eq. (33) also has an $\mathrm{O}(2)$ invariance in the $x y$ plane. This $\mathrm{O}(2)$ symmetry represents the $\mathrm{O}(2)$ nematic symmetry we are going to examine. The relation between the symmetries of the pseudospin model and the microscopic model for spinone bosons has been addressed in previous sections.

In general, the truncation can be applied in the vicinities of all critical points where level crossings between $\mid S, S_{z}$ $=S\rangle$ and $\left|S+2, S_{z}=S+2\right\rangle$ occur, $S+2 \leqslant N$. One arrives at the same phenomenology as for the level crossing between the first two states. Of course, $\epsilon_{0}, \beta$, and $h_{z}$ then depend on the states involved in level crossings and are functions of $S, S$ $=0,2,4, \ldots, N-2$. In the next few subsections we are going to calculate $\epsilon_{0}, \beta$, and $h_{z}$.

\section{B. Calculations of parameters $\epsilon_{0}, \beta$, and $h_{z}$ in the $X X Z$ model}

Microscopic calculations of $\beta$ and $h_{z}$ though straightforward are pretty involved. We present results in a few limits. Detailed calculations can be found in Appendix A.

\section{Two particles per site}

There is only one level crossing in this case. One can verify that

$$
\left\langle\uparrow\left|\hat{Q}_{\alpha \beta}\right| \uparrow\right\rangle=\left(\begin{array}{ccc}
\frac{1}{3} & i & 0 \\
-i & \frac{1}{3} & 0 \\
0 & 0 & -\frac{2}{3}
\end{array}\right),
$$

$$
\left\langle\downarrow\left|\hat{Q}_{\alpha \beta}\right| \uparrow\right\rangle=\left(\begin{array}{ccc}
\frac{1}{\sqrt{3}} & \frac{i}{\sqrt{3}} & 0 \\
\frac{i}{\sqrt{3}} & -\frac{1}{\sqrt{3}} & 0 \\
0 & 0 & 0
\end{array}\right),
$$

and

$$
\left\langle\downarrow\left|\hat{Q}_{\beta \alpha}\right| \uparrow\right\rangle=\left\langle\uparrow\left|\hat{Q}_{\alpha \beta}\right| \downarrow\right\rangle^{\dagger},\left\langle\downarrow\left|\hat{Q}_{\beta \alpha}\right| \downarrow\right\rangle=0 .
$$

Using the Hamiltonian in Eq. (2) and taking into account these matrix elements of $\hat{Q}_{\alpha \beta}$ in the truncated Hilbert space, one obtains the results for $\epsilon_{0}, \beta$, and $h_{z}$. In this particular case, one finds $\epsilon_{0}=4 / 3, \beta=0$ which implies an $\mathrm{O}(3)$ symmetry when the effective field $h_{z}$ vanishes (but with a finite external Zeeman field $H_{z}$ ). It leads to a symmetry higher than the $\mathrm{O}(2)$ one in the original problem in the presence of Zeeman field $H_{z}$.

We believe that the $\mathrm{O}(3)$ symmetry found for two particles per site is accidental and can be removed by taking into account contributions of order of $\eta_{1}=E_{s} / E_{c}$ (see Appendix A for details). The final result can be summarized in the following equation:

$$
\begin{gathered}
\epsilon_{0}=\frac{4}{3}\left(1+\frac{E_{s}}{E_{c}}\right), \beta=-\frac{3 E_{s}}{E_{c}+E_{s}}, \\
h_{z}=-\frac{9 E_{s}-3 H_{z}-8 J_{\mathrm{ex}}-2 \frac{E_{s}}{E_{c}} J_{\mathrm{ex}}}{4 J_{\mathrm{ex}}\left(1+\frac{E_{s}}{E_{c}}\right)} .
\end{gathered}
$$

\section{Four particles per site} that

Close to level crossing between $|0,0\rangle$ and $|2,2\rangle$, we find

$$
\left\langle\uparrow\left|\hat{Q}_{\alpha \beta}\right| \uparrow\right\rangle=\left(\begin{array}{ccc}
\frac{11}{22} & i & 0 \\
-i & \frac{11}{22} & 0 \\
0 & 0 & -\frac{22}{21}
\end{array}\right),
$$

$$
\left\langle\downarrow\left|\hat{Q}_{\alpha \beta}\right| \uparrow\right\rangle=\left(\begin{array}{ccc}
\sqrt{\frac{14}{15}} & i \sqrt{\frac{14}{15}} & 0 \\
i \sqrt{\frac{14}{15}} & -\sqrt{\frac{14}{15}} & 0 \\
0 & 0 & 0
\end{array}\right) .
$$

The corresponding parameters $\epsilon_{0}, \beta$, and $h_{z}$ are 


$$
\epsilon_{0}=\frac{56}{15}, \quad \beta=-\frac{351}{686}, \quad h_{z}=-\frac{15}{56}\left(\frac{3 E_{s}-H_{z}}{J_{\mathrm{ex}}}-\frac{536}{147}\right) .
$$

The effective $X X Z$ model has the desired $\mathrm{O}(2)$ symmetry in the plane perpendicular to the external field. For four particles, level crossing also happens between $|2,2\rangle$ and $|4,4\rangle$ states. Similar calculations have been carried out and presented in Appendix A.

\section{Large-N limit (even $N$ )}

In the large- $N$ limit, one can describe the collective ground state and excited states in terms of spherical harmonics in a quantum rotor representation. So the spin-singlet ground state and polarized $\left|S=2, S_{z}=2\right\rangle$ wave functions are

$$
|\uparrow\rangle=\frac{1}{4} \sqrt{\frac{15}{2 \pi}} \sin ^{2} \theta \exp (i 2 \phi),|\downarrow\rangle=\frac{1}{\sqrt{4 \pi}} .
$$

In the quantum rotor representation, the Hamiltonian is ${ }^{15,10,11,13}$

$$
H=E_{s} \sum_{k} \mathbf{S}_{k}^{2}-H_{z} \sum \mathbf{S}_{z}-J_{\mathrm{ex}} \sum_{\langle k l\rangle}\left[Q_{\alpha \beta}\left(\mathbf{n}_{k}\right) Q_{\beta \alpha}\left(\mathbf{n}_{l}\right)+\text { H.c. }\right],
$$

where $\mathbf{S}=-i \mathbf{n} \times \partial / \partial \mathbf{n}$, the spin operator is defined as the angular momentum of the $\mathrm{O}(3)$ quantum rotor. It is a conjugate variable to director $\mathbf{n}$,

$$
\left[\mathbf{S}_{\alpha}, \mathbf{n}_{\beta}\right]=i \epsilon_{\alpha \beta \gamma} \mathbf{n}_{\gamma} .
$$

Again the matrix elements of $\hat{Q}_{\alpha \beta}$ are calculated below:

$$
\begin{gathered}
\left\langle\uparrow\left|\hat{Q}_{\alpha \beta}\right| \uparrow\right\rangle=\frac{1}{21}\left(\begin{array}{ccc}
2 & 0 & 0 \\
0 & 2 & 0 \\
0 & 0 & -4
\end{array}\right), \\
\left\langle\downarrow\left|\hat{Q}_{\alpha \beta}\right| \uparrow\right\rangle=\frac{1}{\sqrt{30}}\left(\begin{array}{ccc}
1 & i & 0 \\
i & -1 & 0 \\
0 & 0 & 0
\end{array}\right) .
\end{gathered}
$$

The matrix element $\hat{Q}_{x y}$ vanishes in Eq. (44) as an artifact of the large- $N$ approximation. One then obtains all parameters for the $X X Z$ effective model

$$
\epsilon_{0}=\frac{2}{15}, \quad \beta=-\frac{39}{49}, \quad h_{z}=-\frac{15}{2}\left(\frac{3 E_{s}-H_{z}}{J_{\mathrm{ex}}}-\frac{8}{147}\right) .
$$

It is possible to generalize this analysis to level crossing between high-spin states $|S, S\rangle$ and $|S+2, S+2\rangle(S \leqslant N-2)$ and the results are qualitatively the same (see Appendix B). In all cases, $\epsilon_{0}$ is positive and $\beta$ is negative. In the next section, we are going to examine the consequences of this model. Particularly we investigate the implications on magnetically stabilized nematic order and physics around critical points.

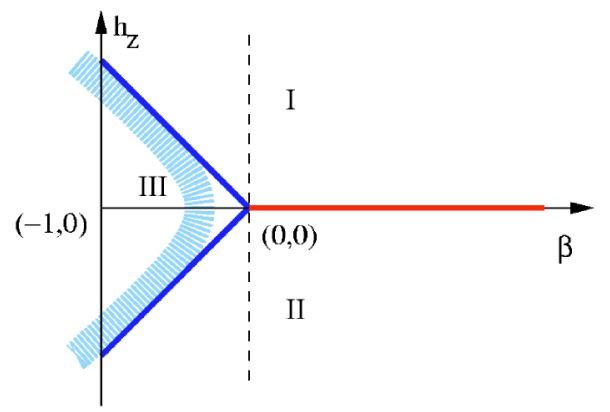

FIG. 2. (Color online) Phases in the ferromagnetic $X X Z$ model. $\beta$ varies from -1 to $+\infty$. Region I, II, and III represent up-polarized (UP), down-polarized (DP), and ferromagnetic ordered (FO) phases, respectively. Along the blue lines $\left(-2 d \beta \pm h_{z}=0\right)$, transitions are continuous while along the red line the transition $\left(h_{z}\right.$ $=0, \beta>0)$ is a first-order one. $\operatorname{Point}(0,0)$ is the $\mathrm{O}(3)$ symmetric tricritical point of the ferromagnetic $X X Z$ model. As $q$ goes to zero, the interactions between magnons are repulsive when $\beta<0$ and attractive when $\beta>0$; along the dash line $(\beta=0)$, magnons are noninteracting. The solutions in the shaded region can be obtained in a dilute gas approximation.

\section{PHASE BOUNDARIES OF THE $X X Z$ MODEL AND HOLSTEIN-PRIMAKOV BOSONS}

\section{A. Phases of $X X Z$ model}

The general phase diagram in the $\left(\beta, h_{z}\right)$ plane can be easily obtained in a mean-field approximation. Later on we argue that the phase boundaries and solutions obtained in this way in some part of the plane are actually exact (see Fig. 2). In the mean-field approximation, we introduce $\mathbf{s}$ as a unit vector order parameter which defines the orientation of spin

$$
\langle\sigma\rangle=2 S \mathrm{~s} .
$$

Here $S=1 / 2$ is the pseudospin.

The $\mathbf{s}$ dependence of the total energy comes entirely from the terms proportional to $\beta$ or $h_{z}$, that is,

$$
\frac{E}{\epsilon_{0} J_{\mathrm{ex}} V_{T}}=\mathrm{const}-4 d \beta S^{2} s_{z}^{2}-2 h_{z} S s_{z},
$$

where $d=3$ is the dimension of three-dimensional cubic lattices. $s_{z}$ varies from -1 and 1 . Minimizing the energy with respect to $\mathbf{S}$ one obtains mean field solutions for various ground states.

Following Eq. (48), when $h_{z}>-2 d \beta$ and $h_{z}>0$, the mean-field solution is

$$
\mathbf{s}=(0,0,1)
$$

representing a fully polarized state which we call the uppolarized (UP) phase. When $h_{z}<2 d \beta$ and $h_{z}<0$, the meanfield solution is

$$
\mathbf{s}=(0,0,-1)
$$

representing another fully polarized state which we call the down-polarized (DP) phase.

In addition, when $-2 d \beta>h_{z}>2 d \beta$ and $\beta<0$, the meanfield solution is 


$$
\mathbf{s}=(\sin \Theta \cos \Phi, \sin \Theta \sin \Phi, \cos \Theta), \quad \cos \Theta=-\frac{h_{z}}{2 d \beta}
$$

representing a ferromagnetically ordered (FO) phase which breaks the in-plane $\mathrm{O}(2)$ symmetry spontaneously. Solutions of different angle $\Phi$ are degenerate and the vacuum manifold is a unit circle $S^{1}$. $\Theta$ varies from $\pi$ to 0 when $h_{z}$ increases from $2 d \beta$ to $-2 d \beta$.

Three phases are separated by a first order transition line along the $h_{z}=0$ axis which starts at point $(0,0)$ and ends at $(\infty, 0)$, and two other second order phase transition lines (see Fig. 2). These two lines are defined by

$$
2 d \beta \pm h_{z}=0
$$

both terminate at point $(0,0)$. Finally $(0,0)$ is a tricritical point.

Along the first order transition line, the UP and DP states become degenerate and the ground state breaks $Z_{2}$ or Ising type of symmetry spontaneously. At the tricritical point $(0,0)$, the $X X Z$ model is $\mathrm{O}(3)$ rotation invariant and the ground state breaks $\mathrm{O}(3)$ symmetry spontaneously. At this point, UP, DP, and FO states are all degenerate.

In UP and DP phases, the microscopic wave functions for ground states are, respectively,

$$
\begin{gathered}
\left|g_{\uparrow}\right\rangle=\prod_{k}|\uparrow\rangle_{k},\left|g_{\downarrow}\right\rangle=\prod_{k}|\downarrow\rangle_{k}, \\
\sigma_{k z}|\uparrow\rangle_{k}=|\uparrow\rangle_{k}, \sigma_{k z}|\downarrow\rangle_{k}=-|\downarrow\rangle_{k} .
\end{gathered}
$$

In the $\mathrm{O}(2)$ ferromagnetic phase

$$
\begin{gathered}
\left|g_{F}\right\rangle=\prod_{k}|\Omega\rangle_{k}, \\
|\Omega\rangle_{k}=\cos \frac{\Theta}{2} \exp (-i \Phi)|\uparrow\rangle_{k}+\sin \frac{\Theta}{2}|\downarrow\rangle_{k}
\end{gathered}
$$

and $\Theta$ is a function of $\beta, h_{z}, \cos \Theta=h_{z} / 2 d \beta,\left|h_{z}\right|<2 d|\beta|$. The solutions in Eq. (54) are degenerate in the $S^{1}$ manifold where $\exp (i \Phi)$ lives and represent spontaneous $\mathrm{O}(2)$-symmetry breaking states.

By examing the microscopic wave functions of UP and DP states, we notice that the UP and DP states are nondegenerate exact eigenstates of the pseudospin operator $\Sigma_{z}$ $=\Sigma_{k} \sigma_{k z}$. Meanwhile, the total pseudospin projected along the $z$ axis is a conserved quantum number. So these UP and DP solutions are exact eigenstates of the $X X Z$ Hamiltonian. In the next subsection we are going to show that they are actually exact ground states when $\beta$ is positive; furthermore we argue that UP or DP states are also exact ground states even when $\beta$ is negative and $h_{z}>-2 d \beta$ or $h_{z}<2 d \beta$.

\section{B. UP and DP states as exact ground states}

When $\beta, h_{z}$ are both positive, the UP state presented above is a ground state of both the $\mathrm{O}(3)$ isotropic term in the ferromagnetic $X X Z$ model and the terms involving $\beta, h_{z}$. So naturally the UP state is the exact ground state of the $X X Z$ model in this limit. Similarly when $\beta$ is positive and $h_{z}$ is negative, the DP state is the exact ground state.

When $\beta<0$ but outside the triangular defined by the two critical lines $2 d \beta \pm h_{z}=0$, we are not able to prove rigorously that eigenstates in Eq. (54) are exact ground states. However, we would like to show that they are locally stable and therefore we argue that they are likely to be the exact ground states.

To carry out this part of discussions, we study the magnon excitation spectrum in UP and DP phases and show that oneparticle magnon excitations are also exact eigenstates; furthermore they are fully gapped except along the second order transition lines. The most straightforward approach to study these excitations is to use the Holstein-Primakov boson representation for the $X X Z$ model.

In the Holstein-Primakov representation, all spin operators are expressed in terms of Holstein-Primakov bosons

$$
\begin{gathered}
\sigma^{+}=\left(\sqrt{2 S-c^{\dagger} c}\right) c, \\
\sigma^{-}=c^{\dagger} \sqrt{2 S-c^{\dagger} c}, \\
\sigma_{z}=2\left(S-c^{\dagger} c\right) .
\end{gathered}
$$

$c^{\dagger}(c)$ is the creation (annihilation) operator of bosons satisfying the usual bosonic commutation relations $\left[c, c^{\dagger}\right]=1$ and the raising and lowering operators are defined as

$$
\sigma^{+}=\frac{\sigma_{x}+i \sigma_{y}}{2}, \quad \sigma^{-}=\frac{\sigma_{x}-i \sigma_{y}}{2} .
$$

One can furthermore verify that

$$
\left[\sigma_{\alpha}, \sigma_{\beta}\right]=i 2 \epsilon_{\alpha \beta \gamma} \sigma^{\gamma}, \quad \sigma \cdot \sigma=4 S(S+1) .
$$

The Hamiltonian of the $X X Z$ model then transforms into

$$
\begin{aligned}
\frac{H_{X X Z}}{\epsilon_{0} J_{\mathrm{ex}}}= & -2 \sum_{\langle k l\rangle} \sqrt{\left[2 S-c_{k}^{\dagger} c_{k}\right]} c_{k} c_{l}^{\dagger} \sqrt{2 S-c_{l}^{\dagger} c_{l}} \\
& -2 \sum_{\langle k l\rangle} c_{k}^{\dagger} \sqrt{\left(2 S-c_{k}^{\dagger} c_{k}\right)\left(2 S-c_{l}^{\dagger} c_{l}\right)} c_{l} \\
& -4(1+\beta) \sum_{\langle k l\rangle} c_{k}^{\dagger} c_{k} c_{l}^{\dagger} c_{l}+2\left[h_{z}+4 S(1+\beta) d\right] \sum_{k} c_{k}^{\dagger} c_{k} .
\end{aligned}
$$

In deriving Eq. (60), we have neglected a constant term $-4(1+\beta) S^{2}-2 h_{z} S$ for each lattice site. In a semiclassical approximation, one indeed recovers the results obtained in the previous section. Again $S=1 / 2$.

To study the excitation spectrum in region I (or II), we first examine the Hamiltonian in Eq. (60) in a one-particle subspace next to the exact eigenstates of UP (or DP). First one notices that a UP state is an exact vacuum for HolsteinPrimakov bosons, that is,

$$
c_{k}\left|g_{\uparrow}\right\rangle=0, \sigma_{k z}\left|g_{\uparrow}\right\rangle=\left|g_{\uparrow}\right\rangle
$$

for any lattice $k$.

One-particle excitations we are interested in live in a subspace of single Holstein-Primakov boson, that is, in a space spanned by states 


$$
c_{k}^{\dagger}\left|g_{\uparrow}\right\rangle
$$

defined at each lattice site $k$. Since the total number operator of Holstein-Primakov bosons commutes with the Hamiltonian

$$
\left[N_{c}, H\right]=0, N_{c}=\sum_{k} c_{k}^{\dagger} c_{k},
$$

$N_{c}$ is a conserved quantum number. We can then diagonalize the Hamiltonian in this one-particle subspace where $N_{c}=1$.

In the subspace, we obtain the following effective Hamiltonian:

$$
\begin{gathered}
\frac{H_{X X Z}^{O P}}{\epsilon_{0} J_{\mathrm{ex}}}=\sum_{\mathbf{q}} \epsilon_{\mathbf{q}} c_{\mathbf{q}}^{\dagger} c_{\mathbf{q}}, \\
\epsilon_{\mathbf{q}}=8 S\left(d-\sum_{\alpha=x, y, z} \cos \mathbf{q}_{\alpha} a\right)+2 h_{z}+8 S d \beta .
\end{gathered}
$$

The superscript OP stands for the "one particle" subspace. Equation (64) indicates the dispersion relation of one-particle states.

In particular, it yields a fully gapped magnon spectrum in region I. The gap vanishes only along the second-order transition line where $\beta<0$ and $h_{z}= \pm 2 d \beta$. Especially magnons are fully gapped along the first-order phase transition line $\beta>0$ and $h_{z}=0$. When $\left|h_{z}\right|<-2 d \beta$ and $\beta<0$, one-particle states (or magnon excitations) start to have lower energies than the vacuum state. This indicates condensation of Holstein-Primakov bosons which we are turning to.

To conclude we find that DP and UP states are exact ground states of the $X X Z$ model in regions I and II. Magnon excitations in these phases are fully gapped. Along the meanfield second-order transition lines, magnons become gapless excitations. Further decreasing $h_{z}$ results in instability of magnon excitations. So we believe that the transition lines in the mean-field theory represent the exact phase boundaries.

\section{Condensation of interacting magnons and emergence of ferromagnetic order in the $X X Z$ model}

As discussed in the Introduction, the dynamics of condensation of magnons depends crucially on the interactions between magnons. To study the region close to critical lines where the condensed particle density should actually be very low, we only take into account two-body interactions and apply a dilute gas expansion. The results we derive in this subsection are valid in the shaded critical regions (see Fig. 2), where

$$
\frac{\left|h_{z} \pm 2 d \beta\right|}{\left|h_{z}\right|} \ll 1 .
$$

And as $h_{z} \pm 2 d \beta$ approaches zero, the results become exact. ${ }^{21}$ We will present the calculations along the upper transition line defined by $h_{z}+2 d \beta=0$; the results are then generalized to the lower transition line $h_{z}-2 d \beta=0$.

In the dilute gas limit which interests us, the number of Holstein-Primakov bosons per lattice site is much less than 1, i.e.,

$$
n_{c}=\left\langle c_{k}^{\dagger} c_{k}\right\rangle \ll 1 .
$$

For this reason, one can expand the nonlinear operators of $\sigma^{ \pm}$ in terms of $n_{c}$, especially,

$$
\sqrt{2 S-c^{\dagger} c}=\sqrt{2 S}\left(1-\frac{c^{\dagger} c}{4 S}+O\left(n_{c}^{2}\right) \cdots\right)
$$

for $S=1 / 2$. The resultant many-body Hamiltonian up to the second order of $n_{c}$ is

$$
\frac{H_{X X Z}}{\epsilon_{0} J_{\mathrm{ex}}}=\sum_{\mathbf{q}} \epsilon_{\mathbf{q}} c_{\mathbf{q}}^{\dagger} c_{\mathbf{q}}-\frac{4 d \beta}{V_{T}} \sum_{\mathbf{q}_{1}, \mathbf{q}_{2}, \mathbf{q}_{3}} c_{\mathbf{q}_{1}+\mathbf{q}_{3}}^{\dagger} c_{\mathbf{q}_{2}-\mathbf{q}_{3}}^{\dagger} c_{\mathbf{q}_{1}} c_{\mathbf{q}_{2}} .,
$$

The first term is identical to $H_{X X Z}^{O P}$, the exact Hamiltonian projected in the one-particle subspace and the second term describes magnon-magnon interactions. This Hamiltonian is applicable in a dilute limit where Eq. (66) is satisfied. The sign of interaction at $q=0$ or small $q$ limit is determined by $\beta$. When $\beta$ is positive, magnon interactions are attractive and when negative magnon interactions are repulsive.

When magnons are ideal $(\beta=0)$, all magnons condense when the energy gap in the spectrum closes at $h_{z}=0$. This leads to an abrupt jump in magnetization which corresponds to the field-driven first-order phase transition from UP to DP phase at the tricritical point $(0,0)$ (along the dashed line). In this case external fields do not induce nematic order. This is consistent with mean-field results discussed in the previous subsection. One can, in principle, generalize this argument to the case when $\beta<0$ and arrive at similar conclusions.

When magnons' interaction are repulsive, following Eq. (68) the chemical potential of magnons in the dilute gas limit (differing from $\mu_{0}$ of atoms) is

$$
\mu=-8 n_{0} d \beta+O\left(n_{0}^{2}\right),
$$

where $n_{0}$ is the number of magnons per lattice site. This is similar to weakly interacting gases of bosons in continuum limit. ${ }^{22}$ The chemical potential defined in this way only depends on intrinsic parameters $\beta$ which have been evaluated microscopically and is independent of external Zeeman fields. The energy of the magnon condensate per lattice site is therefore

$$
\frac{E\left(n_{0}\right)}{\epsilon_{0} J_{\mathrm{ex}} V_{T}}=2\left(\left|h_{z}\right|+2 d \beta\right) n_{0}-4 n_{0}^{2} d \beta .
$$

Minimizing the energy with respect to $n_{0}$ yields

$$
n_{0}=\frac{1}{2}\left[1+\frac{\left|h_{z}\right|}{2 d \beta}\right]
$$

which is a continuous function of $h_{z} \cdot n_{0}$ is much less than one in the critical region where Eq. (66) is satisfied. At the transition point $\left|h_{z}\right|=-2 d \beta$, the magnon density per lattice site either vanishes or is equal to 1 , i.e., $n_{0}=0,1$. Furthermore, if one extrapolates to the $h_{z}=0$ case, one obtains $n_{0}=1 / 2$, that is half magnon per lattice site. $\sigma_{z}=0$ as expected. 
Note that the ground state in this case is not the vacuum of Holstein-Primakov bosons but instead the vacuum defined by Bogolubov quasiparticles. The Bogolubov excitations are created by the following operators:

$$
\begin{gathered}
\gamma_{\mathbf{q}}^{\dagger}=u(\mathbf{q}) c_{\mathbf{q}}^{\dagger}+v(\mathbf{q}) c_{-\mathbf{q}}, \\
u^{2}(\mathbf{q})=\frac{1}{2}\left(1+\frac{\epsilon_{\mathbf{q}}+\mu}{\sqrt{\epsilon_{\mathbf{q}}^{2}+2 \epsilon_{\mathbf{q}} \mu}}\right), \\
v^{2}(\mathbf{q})=-\frac{1}{2}\left(1-\frac{\epsilon_{\mathbf{q}}+\mu}{\sqrt{\epsilon_{\mathbf{q}}^{2}+2 \epsilon_{\mathbf{q}} \mu}}\right),
\end{gathered}
$$

where $\mu$ is the chemical potential of magnons defined before and the kinetic energy $\epsilon_{\mathbf{q}}=2|\mathbf{q}|^{2} a^{2}$ is written in a dimensionless unit. The dispersion of quasiparticles is phononlike $\omega_{q}$ $=v_{s}|\mathbf{q}|$ at small energies, taking into account the chemical potential in Eq. (69), we obtain

$$
v_{s}=v_{s 0} \sqrt{1+\frac{\left|h_{z}\right|}{2 d \beta}}, \quad v_{s 0}=4 a \sqrt{-d \beta} .
$$

This agrees with the semiclassical solutions obtained in Eq. (C12) in Appendix C.

These results indicate that the physics in the $X X Z$ model close to the second-order critical lines is indeed equivalent to interacting dilute magnons defined by Holstein-Primakov bosons. Especially the fact that the emergence of ferromagnetic ordering occurs when condensation of magnons takes place. As in the usual $c$-number approximation for condensed bosons, we approximate

$$
c_{q=0}^{\dagger}=c_{q=0}=\sqrt{n_{0} V_{T}} \exp (i \Phi) .
$$

Substituting this result into the expressions for $\sigma^{ \pm}, \sigma_{z}$ in Eq. (57), we obtain

$$
\begin{gathered}
\left\langle\sigma_{x}\right\rangle=2 \sqrt{2 S} \sqrt{n_{0}} \cos \Phi, \\
\left\langle\sigma_{y}\right\rangle=2 \sqrt{2 S} \sqrt{n_{0}} \sin \Phi, \\
\left\langle\sigma_{z}\right\rangle=2 S-2 n_{0},
\end{gathered}
$$

and again $S=1 / 2$.

Correspondingly, the Bogolubov quasiparticles represent the spin wave excitations in $\mathrm{O}(2)$ ferromagnets. Following Eqs. (57) and (72), one can express $\sigma_{x, y}$ in term of $\gamma^{\dagger}$ and $\gamma$ :

$$
\begin{gathered}
\delta \sigma_{x}(\mathbf{r})=\sum_{\mathbf{q} \neq 0} \exp (i \mathbf{q} \cdot \mathbf{r}) \frac{\sqrt{2 S}}{2 \sqrt{V_{T}}}[u(\mathbf{q})-v(\mathbf{q})]\left(\gamma_{\mathbf{q}}^{\dagger}+\gamma_{-\mathbf{q}}\right), \\
\delta \sigma_{y}(\mathbf{r})=\sum_{\mathbf{q} \neq 0} \exp (i \mathbf{q} \cdot \mathbf{r}) \frac{\sqrt{2 S}}{2 i \sqrt{V_{T}}}[u(\mathbf{q})+v(\mathbf{q})]\left(\gamma_{\mathbf{q}}^{\dagger}-\gamma_{-\mathbf{q}}\right) .
\end{gathered}
$$

Consider a single quasiparticle state

$$
\left|\mathbf{q}_{0}\right\rangle=\gamma_{\mathbf{q}_{0}}^{\dagger}|\mathrm{vac}\rangle .
$$

Spin correlations in this single-particle state are

$$
\begin{gathered}
\left\langle\left[\delta \sigma_{x}(\mathbf{r})-\delta \sigma_{x}(0)\right]^{2}\right\rangle=\frac{2 S}{V_{T}}\left[u\left(\mathbf{q}_{0}\right)-v\left(\mathbf{q}_{0}\right)\right]^{2} \sin ^{2} \frac{\mathbf{q}_{0} \cdot \mathbf{r}}{2}, \\
\left\langle\left[\delta \sigma_{y}(\mathbf{r})-\delta \sigma_{y}(0)\right]^{2}\right\rangle=\frac{2 S}{V_{T}}\left[u\left(\mathbf{q}_{0}\right)+v\left(\mathbf{q}_{0}\right)\right]^{2} \sin ^{2} \frac{\mathbf{q}_{0} \cdot \mathbf{r}}{2}, \\
\left\langle\left[\delta \sigma_{x}(\mathbf{r}) \delta \sigma_{y}(0)\right]\right\rangle=-\frac{S}{V_{T}} \sin \mathbf{q}_{0} \cdot \mathbf{r} .
\end{gathered}
$$

Remarkably, the corresponding orientation of pseudo spin $\mathbf{s}$ derived in the dilute gas approximation is precisely the same as the semiclassical results obtained in Sec. V A; close to the critical line, we notice that

$$
\cos \Theta=1-2 n_{0}, \quad \sin \Theta=2 \sqrt{n_{0}} .
$$

In the next section we are going to discuss the implications of the mapping on correlated atoms, especially magnetically stabilized nematic order. Since the semiclassical solutions turn out to be exact along the critical lines, we would like to believe that solutions are also valid in the ferromagnetic ordered phase, at least qualitatively and can be extrapolated deep into that phase.

\section{NEMATIC ORDER AND PHASE BOUNDARIES OF MAGNETICALLY STABILIZED NEMATIC MOTT STATES}

Let us turn to the problem of Mott states of spin-one bosons. Following discussions in Sec. II, one finds that DP states correspond to spin singlet Mott (SSMI) states and UP states to spin fully polarized Mott (SFPMI) states.

The FO states breaking the $\mathrm{O}(2)$ symmetry represent quantum spin nematic states with easy axis determined by the projection of pseudospin orientation $\mathbf{s}$ in the $x y$ plane. Indeed, for two particles per site the wave function of FO states in Eq. (54) indicates the following spin correlated Mott states for spin one bosons:

$$
\begin{aligned}
\Psi_{N M}= & \prod_{k}\left[\cos \frac{\Theta}{2} \exp \left(-i \frac{\Phi}{2}\right) \frac{\left(\psi_{k x}^{\dagger}+i \psi_{k y}^{\dagger}\right)^{2}}{2 \sqrt{2}}\right. \\
& \left.+\sin \frac{\Theta}{2} \exp \left(i \frac{\Phi}{2}\right) \frac{\psi_{k \alpha}^{\dagger} \psi_{k \alpha}^{\dagger}}{\sqrt{6}}\right]|\mathrm{vac}\rangle .
\end{aligned}
$$

$\Theta$ is a function of $\beta$ and $h_{z}$ as given in Sec. V A,

$$
\cos \frac{\Theta}{2}=\sqrt{\frac{1}{2}-\frac{h_{z}}{4 d \beta}}, \quad \sin \frac{\Theta}{2}=\sqrt{\frac{1}{2}+\frac{h_{z}}{4 d \beta}} .
$$

As $h_{z}$ varies from $-2 d \beta$ to $2 d \beta$, $\Theta$ varies from 0 to $\pi$. And $\Phi \in[0,2 \pi]$ represents an $S^{1}$ manifold for the spontaneous symmetry breaking solutions.

For cold atoms, our calculations show that in all cases $\beta$ is negative. When magnetic fields are varied, the trajectory in the $\beta$ - $h_{z}$ planes (see Fig. 2) moves vertically at a given negative $\beta$. And as magnons are repulsive, magnetic fields stabilize nematic order via the continuous process of condensation of magnons. So as the magnetic field increases, the phases encountered are spin-singlet Mott states, nematic 


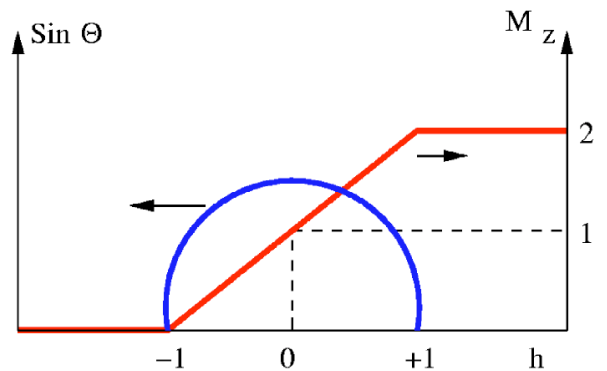

FIG. 3. (Color online) Magnetization $M_{z}$ (in units of $\hbar$ ) and $\sin \Theta$ as a function of $h=h_{z} / 2 d \beta$. sin $\Theta$ defined in Eqs. (51) and (81) is proportional to the nematic order. Results around upper or lower critical points are obtained in a dilute gas approximation and are exact. Notice that the nematic order $\sin \Theta$ reaches the maximum at zero $h_{z}$ where $M_{z}$ is equal to $\hbar$.

Mott states (partially polarized), and spin fully polarized states. Here we will focus on the nematic state.

The projected nematic order parameter for the constructed nematic Mott state is given in Eq. (11) with $\phi=\Phi$ and $\theta$ $=\Theta$ :

$$
\begin{gathered}
Q_{\alpha \beta}^{P x y}=\frac{1}{\sqrt{3}} \sqrt{1-\frac{h_{z}^{2}}{4 d^{2} \beta^{2}}}\left(\omega_{\alpha} \omega_{\beta}-\frac{1}{2} \delta_{\alpha \beta}\right), \\
\omega=\left(\cos \frac{\Phi}{2}, \sin \frac{\Phi}{2}\right) .
\end{gathered}
$$

The nematic order vanishes along the second order critical lines $\left|h_{z}\right|=-2 d \beta$ and reaches maxima when level crossing takes place in an isolated lattice site, i.e., at $h_{z}=0$.

This spin partially polarized nematic Mott state (SPPNMI) has spin polarization

$$
M_{z}=\left\langle\mathbf{S}_{z}\right\rangle=\hbar\left(-\frac{h_{z}}{2 d \beta}+1\right) .
$$

Spins are fully polarized at one of the critical lines $\left(M_{z}\right.$ $=2 \hbar)$ when $h_{z}+2 d \beta=0$ and the spin polarization vanishes $\left(M_{z}=0\right)$ at the other critical line $h_{z}-2 d \beta=0$. In between, $M_{z}$ varies continuously from 0 to $2 \hbar$ and is precisely equal to $\hbar$ when $h_{z}$ vanishes and the nematic order reaches the maximum (see Fig. 3).

Finally, the phase boundaries for SSMI, SFPMI, and SPPNMI can be obtained by substituting the field dependence of $\beta, h_{z}$ derived in Sec. IV B into the expression for critical lines in the $X X Z$ model

$$
h_{z}\left(\frac{E_{s}}{J_{\mathrm{ex}}}, \frac{H_{z}}{J_{\mathrm{ex}}}\right) \pm 2 d \beta\left(\frac{E_{s}}{E_{c}}\right)=0 .
$$

This results in critical fields for various $N$. Especially one determines the upper and lower critical fields $\left(H_{z c}^{ \pm}\right)$between which nematic order develops for the first time when magnetic fields increase from zero.

For $N=2$, the upper and lower critical fields are

$$
H_{z c}^{ \pm}=3 E_{s}-\frac{8}{3} J_{\mathrm{ex}} \pm 24 \frac{E_{s}}{E_{c}} J_{\mathrm{ex}}
$$

and for $N=4$, these fields are

$$
H_{z c}^{ \pm}=3 E_{s}-\frac{536}{147} J_{\mathrm{ex}} \pm \frac{24 \times 117}{245} J_{\mathrm{ex}} .
$$

At the large- $N$ limit, one obtains

$$
H_{z c}^{ \pm}=3 E_{s}-\frac{8}{147} J_{\mathrm{ex}} \pm \frac{156}{245} J_{\mathrm{ex}} .
$$

We have set $d=3$ in deriving Eqs. (85)-(87).

\section{EFFECTS OF QUADRATIC ZEEMAN COUPLING}

In this section, we are going to briefly discuss the effect of quadratic Zeeman terms which generally are present in atomic gases. ${ }^{17,18}$ This kind of external perturbations only conserves the spin projection along the direction of external fields but does not conserve the total spin of the many-body states under consideration and therefore has distinctly different effects on spin-singlet Mott states. Namely, such external fields would induce nematic order at any small but finite coupling. In other words, spin-singlet Mott states are unstable with respect to these perturbations.

To demonstrate this phenomenon, we consider spinsinglet Mott states in the presence of the following quadratic Zeeman perturbation: ${ }^{16,15}$

$$
H_{p}=-H_{Q Z} \sum_{k} \hat{Q}_{\alpha \beta}(k)\left(\mathbf{n}_{\alpha} \mathbf{n}_{\beta}-\frac{1}{3} \delta_{\alpha \beta}\right),
$$

where $\hat{Q}_{\alpha \beta}(k)$ is the nematic operator defined at the beginning of Sec. II [Eq. (2)] and $\mathbf{n}$ characterizes the orientation of quadratic Zeeman fields which we choose to be along the $z$-direction. $H_{Q Z}$ is the strength of the quadratic Zeeman coupling. (This form of perturbation differs slightly from the one used in Refs. 16 and 15 by a singlet operator which does not contribute to the quantity we are calculating here.)

One notices that indeed this quadratic Zeeman term does not communte with the total spin operator defined at any individual lattice site $\hat{\mathbf{S}}_{k}^{2}$; however, it does communte with the operator $\hat{\mathbf{S}}_{k z}$

$$
\begin{gathered}
{\left[\hat{\mathbf{S}}_{k z}, \sum_{k^{\prime}} \hat{Q}_{\alpha^{\prime} \beta^{\prime}}\left(k^{\prime}\right) \delta_{\alpha^{\prime} z} \delta_{\beta^{\prime} z}\right]=0,} \\
\hat{\mathbf{S}}_{k z}=-i \sum_{k} \epsilon_{z \alpha \beta} \psi_{k \alpha}^{\dagger} \psi_{k \beta} .
\end{gathered}
$$

So what it does is to cause transitions between states with different on-site spin quantum numbers $S_{k}$ but with identical spin projection along the $z$ direction $\mathbf{S}_{k z}$. Thus it does not lead to transitions between different $\mathbf{S}_{k z}$ subspaces. For a spin singlet Mott state, this perturbation results in transitions between on-site singlet states $\left(S_{k}=0\right)$ and nonsinglet states $\left(S_{k} \neq 0\right)$ in the subspace of $\mathbf{S}_{k z}=0$.

For two particles per site and in the zero hopping limit, we find that these transitions lead to coherent superposition 
of two states in $S_{z}=0$ subspace: $|0.0\rangle$ state and

$$
|2,0\rangle=\frac{1}{2 \sqrt{3}}\left(3 \psi_{z}^{\dagger} \psi_{z}^{\dagger}-\psi_{\alpha}^{\dagger} \psi_{\alpha}^{\dagger}\right)|\mathrm{vac}\rangle, \quad \alpha=x, y, z .
$$

For instance, in the first-order perturbation expansion the ground-state wave function is

$$
\begin{aligned}
\delta \Psi= & \frac{\sqrt{2} H_{Q Z}}{9 E_{s}} \sum_{k} \frac{1}{2 \sqrt{3}}\left(3 \psi_{k z}^{\dagger} \psi_{k z}^{\dagger}-\psi_{k \eta}^{\dagger} \psi_{k \eta}^{\dagger}\right) \\
& \otimes \prod_{l \neq k} \frac{1}{\sqrt{6}} \psi_{l \eta^{\prime}}^{\dagger} \psi_{l \eta^{\prime}}^{\dagger}|\mathrm{vac}\rangle .
\end{aligned}
$$

A direct calculation shows that the nematic order is induced continuously as the quadratic coupling is applied

$$
\left\langle\hat{Q}_{\alpha \beta}\right\rangle=\frac{2}{3} \frac{H_{Q Z}}{E_{s}}\left(\mathbf{n}_{\alpha} \mathbf{n}_{\beta}-\frac{1}{3} \delta_{\alpha \beta}\right) .
$$

This dependence is very different from the linear-Zeeman field dependence of nematic order which exhibits a critical field below which spin singlet Mott states are stable. As expected, quadratic Zeeman effects are more effective in stabilizing spin nematic Mott states. Furthermore, the easy axis of the induced nematic tensor order parameter is pinned along the direction of external fields, $\mathbf{n}$ and the resultant states are Ising nematically ordered instead of $\mathrm{O}(2)$ or $\mathrm{O}(3)$ nematic states discussed before.

\section{CONCLUSIONS}

To summarize, in this article we have investigated magnetically stabilized fluctuating spin nematic order. We have shown that nematic order can develop when two nonnematic states at a lattice site are in a linear superposition in the presence of external fields. When external fields are applied, even small superexchange coupling could lead to such a linear superposition and nematic order emerges even though no spontaneous symmetry breaking occurs in zero field.

We have also mapped the problem of spin-one bosons with antiferromagnetic interactions in an external field to the ferromagnetic $X X Z$ spin $(S=1 / 2)$ model. We find that the field-driven quantum phase transitions belong to the universality class of the ferromagnetic $X X Z$ model $(S=1 / 2)$. Spontaneous symmetry breaking in the $x y$ plane in this effective ferromagnetic $X X Z$ model corresponds to planar nematic ordering in the underlying atomic states. In all non-nematic Mott states which interest us, interactions between magnons are repulsive. Therefore when the external field reaches a critical one, condensation and thus phase transitions are continuous.

We also show that the breaking of the U(1) symmetry in magnon Bose condensates results in breaking of the $\mathrm{O}(2)$ nematic symmetry in the $x y$ plane perpendicular to external fields. The Bogoliubov quasiparticles of condensates are precisely the spin wave excitations in the $\mathrm{O}(2)$ nematic states. So the nematic order is stabilized when the field exceeds a critical one and magnons condense. We have also obtained the microscopic wave functions of ordered states and spinwave excitations.

Finally we find that for a spin-singlet Mott state the fluctuating nematic order can be stabilized by any small but finite quadratic Zeeman effects. Namely, the nematic order parameter varies continuously in the presence of quadratic Zeeman effects.

\section{ACKNOWLEDGMENTS}

F.Z. would like to thank P. Wiegmann for useful discussions about fermionization of magnons; he also would like to thank KITP, UCSB for its hospitality during the workshop on Quantum gases in 2004. M.S. and J.W. were supported by the FOM (Netherlands) under Contract 02SIC25 and NWO-MK "Projectruimte" Grant No. 00PR1929. This project is also in part supported by the U.S. National Science Foundation Under Grant No. PHY 99-07949 (F.Z.), a research Grant from UBC (F.Z.) and NSERC (F.Z., I.A.) and CIAR (I.A.).

\section{APPENDIX A: EFFECTIVE $X X Z$ MODEL FOR VARIOUS NUMBERS OF PARTICLES PER SITE}

\section{Microscopic Hamiltonian}

To break the $\mathrm{O}(3)$ symmetry in the $X X Z$ model for two particles, we keep higher order terms of $o\left(E_{s} / E_{c}\right)$. The effective Hamiltonian for spin-one bosons in the Mott state of an optical lattice in the presence of a magnetic field (in the $z$ direction) can be derived as

$$
\begin{aligned}
H= & \left(E_{s}-6 J_{\mathrm{ex}} \frac{E_{s}}{E_{c}}\right) \sum_{k} \hat{\mathbf{S}}_{k}^{2}-H_{z} \sum_{k} \hat{\mathbf{S}}_{k}^{z} \\
& -2 J_{\mathrm{ex}}\left(1+\frac{E_{s}}{E_{c}}\right) \sum_{\langle k l\rangle} \hat{Q}_{k, \alpha \beta} \hat{Q}_{l, \beta \alpha} \\
& +2 J_{\mathrm{ex}} \frac{E_{s}}{E_{c}} \sum_{\langle k l\rangle}\left(\hat{\mathbf{S}}_{k}+\hat{\mathbf{S}}_{l}\right)^{2} \\
& +J_{e x} \frac{E_{s}}{E_{c}\langle k l\rangle} \operatorname{Tr}[\mathcal{A}(k) \mathcal{N}(l)+\mathcal{A}(l) \mathcal{N}(k)] .
\end{aligned}
$$

We have introduced the operators $\mathcal{N}_{\eta \xi}=\psi_{\eta}^{\dagger} \psi_{\xi}$ and $\mathcal{A}_{\eta \xi}$ $=\psi_{\eta}^{\dagger} \psi_{\xi}^{\dagger} \psi_{\beta} \psi_{\beta}-\psi_{\alpha}^{\dagger} \psi_{\alpha}^{\dagger} \psi_{\eta} \psi_{\xi}$

\section{2. $N=2$ case}

For $N=2$ we have only the possibility of looking at the transition between the states $|\uparrow\rangle=|2,2\rangle$ and $|\downarrow\rangle=|0,0\rangle$. The relevant nonvanishing matrix elements are (again $\left.\left\langle\uparrow\left|\hat{Q}_{\alpha \beta}\right| \downarrow\right\rangle=\left\langle\uparrow\left|\hat{Q}_{\alpha \beta}\right| \uparrow\right\rangle^{\dagger}\right)$

$$
\left\langle\uparrow\left|\hat{Q}_{\alpha \beta}\right| \uparrow\right\rangle=\left(\begin{array}{ccc}
\frac{1}{3} & i & 0 \\
-i & \frac{1}{3} & 0 \\
0 & 0 & -\frac{2}{3}
\end{array}\right),
$$




$$
\left\langle\downarrow\left|\hat{Q}_{\alpha \beta}\right| \uparrow\right\rangle=\left(\begin{array}{ccc}
\frac{1}{\sqrt{3}} & \frac{i}{\sqrt{3}} & 0 \\
\frac{i}{\sqrt{3}} & -\frac{1}{\sqrt{3}} & 0 \\
0 & 0 & 0
\end{array}\right), \quad(\mathrm{A} 4) \quad h_{z}=-\frac{9 E_{s}-3 H_{z}-8 J_{\mathrm{ex}}-2 \frac{E_{s}}{E_{c}} J_{\mathrm{ex}}}{4 J_{\mathrm{ex}}\left(1+\frac{E_{s}}{E_{c}}\right)}
$$$$
\langle\uparrow|\mathcal{N}| \uparrow\rangle=\frac{1}{\sqrt{3}}\left(\begin{array}{ccc}
1 & i & 0 \\
-i & 1 & 0 \\
0 & 0 & 0
\end{array}\right),
$$$$
\langle\uparrow|\mathcal{N}| \downarrow\rangle=\frac{1}{\sqrt{3}}\left(\begin{array}{ccc}
1 & -i & 0 \\
-i & -1 & 0 \\
0 & 0 & 0
\end{array}\right),
$$$$
\langle\downarrow|\mathcal{N}| \uparrow\rangle=\frac{1}{\sqrt{3}}\left(\begin{array}{ccc}
1 & i & 0 \\
i & -1 & 0 \\
0 & 0 & 0
\end{array}\right),
$$$$
\langle\downarrow|\mathcal{N}| \downarrow\rangle=\frac{2}{3}\left(\begin{array}{ccc}
1 & 0 & 0 \\
0 & 1 & 0 \\
0 & 0 & 1
\end{array}\right) .
$$$$
\langle\uparrow|\mathcal{A}| \downarrow\rangle=\sqrt{3}\left(\begin{array}{ccc}
1 & -i & 0 \\
-i & -1 & 0 \\
0 & 0 & 0
\end{array}\right),
$$$$
\langle\downarrow|\mathcal{A}| \uparrow\rangle=\sqrt{3}\left(\begin{array}{ccc}
-1 & -i & 0 \\
-i & 1 & 0 \\
0 & 0 & 0
\end{array}\right) .
$$

Using these results we find that the effective Hamiltonian turns out to be

$$
\begin{aligned}
H= & -J_{\mathrm{ex}} \frac{4}{3}\left(1+\frac{E_{s}}{E_{c}}\right) \sum_{\langle k l\rangle} \sigma_{k} \cdot \sigma_{l}+4 J_{\mathrm{ex}} \frac{E_{s}}{E_{c}} \sum_{\langle k l\rangle} \sigma_{k}^{z} \sigma_{l}^{z} \\
& -\left(H_{z}-3 E_{s}+\frac{8}{3} J_{\mathrm{ex}}+\frac{2}{3} \frac{E_{s}}{E_{c}} J_{\mathrm{ex}}\right) \sum_{k} \sigma_{k}^{z} .
\end{aligned}
$$

This is the Hamiltonian for the $X X Z$ model:

$$
\frac{H_{X X Z}}{\epsilon_{0} J_{\mathrm{ex}}}=-\sum_{\langle k l\rangle} \sigma_{k} \cdot \sigma_{l}-\beta \sum_{\langle k l\rangle} \sigma_{k}^{z} \sigma_{l}^{z}-h_{z} \sum_{k} \sigma_{k}^{z}
$$

with

$$
\begin{gathered}
\epsilon_{0}=\frac{4}{3}\left(1+\frac{E_{s}}{E_{c}}\right), \\
\beta=-\frac{3 E_{s}}{E_{c}+E_{s}},
\end{gathered}
$$

\section{3. $N=4$ case}

For four particles per site, there are two possible transitions: $|0,0\rangle \rightarrow|2,2\rangle \rightarrow|4,4\rangle$. We will consider both transitions. In both cases the correction of order $J_{\text {ex }}\left(E_{s} / E_{c}\right)$ turns out to be not particularly interesting.

$$
\text { a. }|0,0\rangle \rightarrow|2,2\rangle
$$

We define again $|\uparrow\rangle=|2,2\rangle$ and $|\downarrow\rangle=|0,0\rangle$. The relevant nonvanishing matrix elements are

$$
\left\langle\uparrow\left|\hat{Q}_{\alpha \beta}\right| \uparrow\right\rangle=\left(\begin{array}{ccc}
\frac{11}{21} & i & 0 \\
-i & \frac{11}{21} & 0 \\
0 & 0 & -\frac{22}{21}
\end{array}\right),
$$

This gives rise to an $X X Z$ model with the following parameters

$$
\epsilon_{0}=\frac{56}{15}
$$

$$
\begin{gathered}
\beta=-\frac{351}{686}, \\
h_{z}=-\frac{1}{\epsilon_{0} J_{\mathrm{ex}}}\left(3 E_{s}-H_{z}-\frac{536}{147} J_{\mathrm{ex}}\right) .
\end{gathered}
$$

$$
\text { b. }|2,2\rangle \rightarrow|4,4\rangle
$$

We define $|\uparrow\rangle=|4,4\rangle$ and $|\downarrow\rangle=|2,2\rangle$. The relevant nonvanishing matrix elements are

$$
\left\langle\uparrow\left|\hat{Q}_{\alpha \beta}\right| \uparrow\right\rangle=\left(\begin{array}{ccc}
\frac{2}{3} & 2 i & 0 \\
-2 i & \frac{2}{3} & 0 \\
0 & 0 & -\frac{4}{3}
\end{array}\right),
$$




$$
\begin{aligned}
& \left\langle\downarrow\left|\hat{Q}_{\alpha \beta}\right| \uparrow\right\rangle=\left(\begin{array}{ccc}
\sqrt{\frac{12}{7}} & \sqrt{\frac{12}{7}} i & 0 \\
\sqrt{\frac{12}{7}} i & -\sqrt{\frac{12}{7}} & 0 \\
0 & 0 & 0
\end{array}\right), \\
& \left\langle\downarrow\left|\hat{Q}_{\alpha \beta}\right| \downarrow\right\rangle=\left(\begin{array}{ccc}
\frac{11}{21} & i & 0 \\
-i & \frac{11}{21} & 0 \\
0 & 0 & -\frac{22}{21}
\end{array}\right) .
\end{aligned}
$$

Using this we get again an effective Hamiltonian in the form of an $X X Z$ model. The parameters are

$$
\begin{gathered}
\epsilon_{0}=\frac{48}{7}, \\
\beta=-\frac{284}{49} \frac{1}{\epsilon_{0}}=-\frac{71}{84}, \\
h_{z}=-\frac{1}{\epsilon_{0} J_{\mathrm{ex}}}\left(7 E_{s}-H_{z}-\frac{344}{49} J_{\mathrm{ex}}\right) .
\end{gathered}
$$

\section{4. $N=3$ case}

Although in this article we only treat the case with even numbers of particles per site, the approach developed to study the physics close to critical fields can also be applied to the case with odd numbers of particles per site. For instance, for three particles per site we have the transition between $|\uparrow\rangle=|3,3\rangle$ and $|\downarrow\rangle=|1,1\rangle$. The nonvanishing matrix elements are

$$
\begin{aligned}
& \left\langle\uparrow\left|\hat{Q}_{\alpha \beta}\right| \uparrow\right\rangle=\left(\begin{array}{ccc}
\frac{1}{2} & \frac{3}{2} i & 0 \\
-\frac{3}{2} i & \frac{1}{2} & 0 \\
0 & 0 & -1
\end{array}\right), \\
& \left\langle\downarrow\left|\hat{Q}_{\alpha \beta}\right| \uparrow\right\rangle=\left(\begin{array}{ccc}
\sqrt{\frac{3}{5}} & \sqrt{\frac{3}{5}} i & 0 \\
\sqrt{\frac{3}{5}} i & -\sqrt{\frac{3}{5}} & 0 \\
0 & 0 & 0
\end{array}\right),
\end{aligned}
$$

$$
\left\langle\downarrow\left|\hat{Q}_{\alpha \beta}\right| \downarrow\right\rangle=\left(\begin{array}{ccc}
\frac{3}{10} & \frac{i}{2} & 0 \\
-\frac{i}{2} & \frac{3}{10} & 0 \\
0 & 0 & -\frac{3}{5}
\end{array}\right) .
$$

The parameters of the $X X Z$ model in this case are

$$
\begin{aligned}
& \epsilon_{0}=\frac{12}{5}, \\
& \beta=-\frac{8}{15},
\end{aligned}
$$

$$
h_{z}=-\frac{1}{\epsilon_{0} J_{\mathrm{ex}}}\left(5 E_{s}-H_{z}-\frac{124}{25} J_{\mathrm{ex}}\right) .
$$

\section{APPENDIX B: EFFECTIVE HAMILTONIAN FOR LARGE $N$}

For large $N, N$ even, we can study all transitions from $|S, S\rangle \rightarrow|S+2, S+2\rangle$. These states are given by

$$
|S, S\rangle=\left|Y_{S S}(\mathbf{n})\right\rangle=(-1)^{S} \sqrt{\frac{2 S+1}{4 \pi} \frac{(2 S) !}{2^{2 S}(S !)^{2}}} e^{i S \phi} \sin ^{S} \theta .
$$

The hopping term in the Hamiltonian is just equal to

$$
-2 J_{\mathrm{ex}} \sum_{\langle k l\rangle}\left(\mathbf{n}_{k} \cdot \mathbf{n}_{l}\right)^{2} .
$$

Introducing $|\uparrow\rangle=|S+2, S+2\rangle$ and $|\downarrow\rangle=|S, S\rangle$, we get the following nonvanishing matrix elements

$$
\begin{gathered}
\left\langle\left.\uparrow\right|_{k}\left\langle\left.\uparrow\right|_{l}\left(\mathbf{n}_{k} \cdot \mathbf{n}_{l}\right)^{2} \mid \uparrow\right\rangle_{k} \mid \uparrow\right\rangle_{l}=\frac{19+12 S+2 S^{2}}{(7+2 S)^{2}}, \\
\left\langle\left.\uparrow\right|_{k}\left\langle\left.\downarrow\right|_{l}\left(\mathbf{n}_{k} \cdot \mathbf{n}_{l}\right)^{2} \mid \uparrow\right\rangle_{k} \mid \downarrow\right\rangle_{l}=\frac{7+8 S+2 S^{2}}{(3+2 S)(7+2 S)}, \\
\left\langle\left.\uparrow\right|_{k}\left\langle\left.\downarrow\right|_{l}\left(\mathbf{n}_{k} \cdot \mathbf{n}_{l}\right)^{2} \mid \downarrow\right\rangle_{k} \mid \uparrow\right\rangle_{l}=\frac{(2+S)(1+S)}{(3+2 S)(5+2 S)}, \\
\left\langle\left.\downarrow\right|_{k}\left\langle\left.\downarrow\right|_{l}\left(\mathbf{n}_{k} \cdot \mathbf{n}_{l}\right)^{2} \mid \downarrow\right\rangle_{k} \mid \downarrow\right\rangle_{l}=\frac{3+4 S+2 S^{2}}{(3+2 S)^{2}} .
\end{gathered}
$$

The effective Hamiltonian turns out to be 


$$
\begin{aligned}
\frac{H_{\mathrm{eff}}}{J_{\mathrm{ex}}}= & -\sum_{\langle k l\rangle} \frac{(2+S)(1+S)}{(3+2 S)(5+2 S)}\left(\sigma_{k}^{x} \sigma_{l}^{x}+\sigma_{k}^{y} \sigma_{l}^{y}\right) \\
& -\sum_{\langle k l\rangle} \frac{12}{(3+2 S)^{2}(7+2 S)^{2}} \sigma_{k}^{z} \sigma_{l}^{z} \\
& -\left((2 S+3) \frac{E_{s}}{J_{\mathrm{ex}}}-\frac{B_{z}}{J_{\mathrm{ex}}}-\frac{8(3+S)(1+2 S)}{(3+2 S)^{2}(7+2 S)^{2}}\right) \sum_{k} \sigma_{k}^{z} .
\end{aligned}
$$

This is clearly an $X X Z$ model with

$$
\begin{gathered}
\epsilon_{0}=\frac{(2+S)(1+S)}{(3+2 S)(5+2 S)}, \\
\beta=-1+\frac{12(5+2 S)}{\left[(7+2 S)^{2}\left(6+13 S+9 S^{2}+2 S^{3}\right)\right]}, \\
h_{z}=-\frac{1}{\epsilon_{0} J_{\mathrm{ex}}}\left((2 S+3) E_{s}-B_{z}-\frac{8(3+S)(1+2 S)}{(3+2 S)^{2}(7+2 S)^{2}} J_{\mathrm{ex}}\right) .
\end{gathered}
$$

It is clear that $\beta$ is negative for all $S$ and it gets more negative if we increase $S$. For the lowest transition $|0,0\rangle$ $\rightarrow|2,2\rangle$ this gives the numbers

$$
\begin{gathered}
\epsilon_{0}=\frac{2}{15}, \\
\beta=-\frac{39}{49}, \\
h_{z}=-\frac{1}{\epsilon_{0} J_{\mathrm{ex}}}\left(3 E_{s}-H_{z}-\frac{8}{147} J_{\mathrm{ex}}\right) .
\end{gathered}
$$

\section{APPENDIX C: THE HOLSTEIN-PRIMAKOV BOSONS REPRESENTATION}

\section{Holstein-Primakov bosons in UP or DP phases}

The Hamiltonian of the $X X Z$ model is given as

$$
\frac{H_{X X Z}}{\epsilon_{0} J_{\mathrm{ex}}}=-\sum_{\langle k l\rangle} \sigma_{k \alpha} \sigma_{l \alpha}-\beta \sum_{\langle k l\rangle} \sigma_{k z} \sigma_{l z}-h_{z} \sum_{k} \sigma_{k z} .
$$

In this subsection, we are interested in region I (see Fig. 2 ), where $h_{z}+2 d \beta>0$. After Fourier transforming and in terms of Holstein-Primakav (HP) bosons the Hamiltonian can written as

$$
H_{X X Z}=H^{(0)}+H^{(2)}+H^{(4)}+\mathcal{O}\left[\left(\hat{c}^{(\dagger)}\right)^{6}\right] .
$$

Here

$$
\begin{gathered}
H^{(0)}=-V^{T}\left[d(\beta+1)+h_{z}\right], \\
H^{(2)}=\sum_{\mathbf{q}}\left[4(1+\beta) d+2 h_{z}-4 \sum_{\alpha} \cos \left(q_{\alpha} a\right)\right] c_{\mathbf{q}}^{\dagger} c_{\mathbf{q}},
\end{gathered}
$$

and the fourth order term is

$$
\begin{aligned}
& \frac{H^{(4)}}{J_{\mathrm{ex}} \epsilon_{0}}=\frac{1}{V_{T \mathbf{q}_{1} \mathbf{q}_{2} \mathbf{q}_{3}}} c_{\mathbf{q}_{1}}^{\dagger} c_{\mathbf{q}_{2}}^{\dagger} c_{\mathbf{q}_{3}} c_{\mathbf{q}_{1}+\mathbf{q}_{2}-\mathbf{q}_{3}} \sum_{\alpha}\left\{\exp \left(-i \mathbf{q}_{2 \alpha} a\right)\right. \\
& +\exp \left(-i \mathbf{q}_{3 \alpha} a\right)+\exp \left[-i\left(-\mathbf{q}_{1}-\mathbf{q}_{2}+\mathbf{q}_{3}\right)_{\alpha} a\right] \\
& \left.+\exp \left(i \mathbf{q}_{1 \alpha} a\right)\right\}-4(1+\beta) \sum_{\mathbf{q}_{1} \mathbf{q}_{2} \mathbf{q}_{3}} c_{\mathbf{q}_{1}}^{\dagger} c_{\mathbf{q}_{2}}^{\dagger} c_{\mathbf{q}_{3}} c_{\mathbf{q}_{1}+\mathbf{q}_{2}-\mathbf{q}_{3}} \\
& \times \sum_{\alpha} \exp \left[i\left(\mathbf{q}_{1}-\mathbf{q}_{3}\right)_{\alpha} a\right] \text {. }
\end{aligned}
$$

Here $a$ is the lattice constant.

Following Eq. (C4), the energy of the quasiparticles is given by

$$
\epsilon_{\mathbf{q}}=4(1+\beta) d-4 \sum_{\alpha=x, y, z} \cos \left(\mathbf{q}_{\alpha} a\right)+2 h_{z},
$$

where the energy gap in the spectrum is given as

$$
\Delta\left(\beta, h_{z}\right)=4 \beta d+2 h_{z} .
$$

The fourth order term describes interactions between magnons. Indeed, in the small $|\mathbf{q}|$ limit the Hamiltonian can approximately be written as (up to a constant)

$$
\frac{H_{X X Z}}{J_{\mathrm{ex}} \epsilon_{0}}=\sum_{\mathbf{k}} \epsilon_{\mathbf{q}} c_{\mathbf{q}}^{\dagger} c_{\mathbf{q}}-\frac{4 d \beta}{V_{T}} \sum_{\mathbf{q}_{1} \mathbf{q}_{2} \mathbf{q}_{3}} c_{\mathbf{q}_{1}}^{\dagger} c_{\mathbf{q}_{2}}^{\dagger} c_{\mathbf{q}_{3}} c_{\mathbf{q}_{1}+\mathbf{q}_{2}-\mathbf{q}_{3}} .
$$

When $\beta>0$ interactions between the magnons are attractive and when $\beta<0$ interactions are repulsive. To derive these results, we have used the dilute gas expansion expansion

$$
\sqrt{2 S-c^{\dagger} c}=\left(1-\frac{c^{\dagger} c}{2}-\frac{1}{8}\left(c^{\dagger} c\right)^{2}+\cdots\right) .
$$

\section{Holstein-Primakov bosons in ferromagnetically ordered phase}

The most convenient way to study HP bosons in region III is to introduce the following rotation:

$$
\left(\begin{array}{l}
x \\
y \\
z
\end{array}\right)=\left(\begin{array}{c}
\cos \Theta x^{\prime}+\sin \Theta z^{\prime} \\
y^{\prime} \\
\cos \Theta x^{\prime}-\sin \Theta z^{\prime}
\end{array}\right) .
$$

In the semiclassical approximation, by minimizing the energy with respect to $\Theta$, one obtains the ground-state solution with $\cos \Theta=-h_{z} / 2 d \beta$.

Consider an expansion over this solution. We get the following lowest order terms:

$$
\begin{aligned}
\frac{H_{X X Z}^{(2)}}{\epsilon_{0} J_{\mathrm{ex}}}= & \sum_{\mathbf{q}}\left(4 d+4 d \beta \cos ^{2} \Theta+2 h_{z} \cos \Theta\right) c_{\mathbf{q}}^{\dagger} c_{\mathbf{q}} \\
& -\sum_{\mathbf{q}}\left(4+2 \beta \sin ^{2} \Theta\right) \sum_{\alpha} \cos \mathbf{q}_{\alpha} a c_{\mathbf{q}}^{\dagger} c_{\mathbf{q}} \\
& -\sum_{\mathbf{q}} \beta \sin ^{2} \Theta \sum_{\alpha} \cos \mathbf{q}_{\alpha} a\left(c_{\mathbf{q}} c_{\mathbf{q}}+c_{\mathbf{q}}^{\dagger} c_{\mathbf{q}}^{\dagger}\right) .
\end{aligned}
$$

When $\Theta=0$, one recovers the results in Sec. V B. 
Taking into account $\cos \Theta=-h_{z} / 2 d \beta$ in the ferromagnetic phase, in the long wave length limit one further simplies the result to

$$
\begin{aligned}
\frac{H_{X X Z}^{(2)}}{\epsilon_{0} J_{\mathrm{ex}}}= & \sum_{\mathbf{q}} 4\left(d-\sum_{\alpha} \cos \mathbf{q}_{\alpha} a\right) c_{\mathbf{q}}^{\dagger} c_{\mathbf{q}}-\sum_{\mathbf{q}}\left(2 d \beta \sin ^{2} \Theta\right) c_{\mathbf{q}}^{\dagger} c_{\mathbf{q}} \\
& -\sum_{\mathbf{q}} d \beta \sin ^{2} \Theta\left(c_{\mathbf{q}} c_{\mathbf{q}}+c_{\mathbf{q}}^{\dagger} c_{\mathbf{q}}^{\dagger}\right) .
\end{aligned}
$$

This yields the following dispersion:

$$
\omega_{\mathbf{q}}=2 \sqrt{2} a \sqrt{-d \beta} \sqrt{1-\frac{h_{z}^{2}}{4 d^{2} \beta^{2}}}|\mathbf{q}| .
$$

Equation (C12) agrees with the results derived in the dilute gas approximation in $\underline{\mathrm{Sec}}$. V C; close to critical lines, one notices that $\sin \Theta=2 \sqrt{n_{0}}$ and $d \beta \sin ^{2} \Theta=4 d \beta n_{0}$.
${ }^{1}$ F. D. M. Haldane, Phys. Rev. Lett. 50, 1153 (1983).

${ }^{2}$ I. Affleck, T. Kennedy, E. H. Lieb, and H. Tasaki, Phys. Rev. Lett. 59, 799 (1987).

${ }^{3}$ D. P. Arovas, A. Auerbach, and F. D. M. Haldane, Phys. Rev. Lett. 60, 531 (1988).

${ }^{4}$ I. Affleck, Phys. Rev. B 43, 3215 (1991).

${ }^{5}$ In one-dimensions, a Majorana fermion representation was employed to study magnetization in A. M. Tsvelik, Phys. Rev. B 42, 10499 (1990).

${ }^{6}$ K. Nomura and T. Sakai, Phys. Rev. B 44, 5092 (1991); T. Sakai and M. Takahashi, ibid. 43, 13383 (1991).

${ }^{7}$ E. S. Sorensen and I. Affleck, Phys. Rev. Lett. 71, 1633 (1993).

${ }^{8}$ T. Nikuni, M. Oshikawa, A. Oosawa, and H. Tanaka, Phys. Rev. Lett. 84, 5868 (2000).

${ }^{9}$ G. Mishguich and M. Oshikawa, cond-mat/0405422.

${ }^{10}$ E. Demler and F. Zhou, Phys. Rev. Lett. 88, 163001 (2002); E. Demler, F. Zhou, and D. F. M. Haldane (unpublished).

${ }^{11}$ F. Zhou and M. Snoek, Ann. Phys. (N.Y.) 308, 692 (2003).

${ }^{12}$ A. Imambekov, M. Lukin, and E. Demler, Phys. Rev. A 68, 063602 (2003).

${ }^{13}$ M. Snoek and F. Zhou, Phys. Rev. B 69, 094410 (2004).

${ }^{14}$ Fei Zhou, Europhys. Lett. 63(4), 505 (2003).

${ }^{15}$ F. Zhou, Phys. Rev. Lett. 87, 080401 (2001); F. Zhou, Int. J.
Mod. Phys. B 17, 2643 (2003).

${ }^{16}$ J. Wiemer and F. Zhou, Phys. Rev. B 70, 115110 (2004).

${ }^{17}$ T. L. Ho, Phys. Rev. Lett. 81, 742 (1998); C. K. Law, H. Pu and N. Bigelow, ibid. 81, 5257 (1998); T. Ohmi and K. Machida, J. Phys. Soc. Jpn. 67, 1822 (1998). Discussions about the quadratic Zeeman effects can be found in T. L. Ho and S. Yip, Phys. Rev. Lett. 84, 4031 (2000).

${ }^{18}$ For experiments on spinor condensates, see D. M. Stamper-Kurn, M. R. Andrews, A. P. Chikkatur, S. Inouye, H.-J. Miesner, J. Stenger, and W. Ketterle, Phys. Rev. Lett. 80, 2027 (1998); J. Stenger, S. Inouye, D. M. Stamper-Kurn, H.-J. Miesner, A. P. Chikkatur, and W. Ketterle, Nature (London) 396, 345 (1998).

${ }^{19}$ A. Imambekov, M. Lukin, and E. Demler, Phys. Rev. Lett. 93, 120405 (2004).

${ }^{20}$ This traceless projected order parameter differs from the characterization discussed in E. Mueller, Phys. Rev. A 69, 033606 (2004).

${ }^{21}$ The dilute gas approximation applies only in high-dimensional lattices. In one dimension, fermionization is needed and this subject will be examined in a subsequent work.

${ }^{22}$ P. Nozieres and D. Pines, The theory of Quantum Liquids, Vol II: Superfluid Bose Liquids (Addison-Wesley, New York, 1990). 